\title{
UDP-glucose and $\mathrm{P}_{2} \mathrm{Y}_{14}$ receptor amplify allergen-induced airway eosinophilia
}

\author{
Tadeusz P. Karcz, ${ }^{1}$ Gregory S. Whitehead, ${ }^{1}$ Keiko Nakano, ${ }^{1}$ Hideki Nakano, ${ }^{1}$ Sara A. Grimm, ${ }^{2}$ Jason G. Williams, ${ }^{3}$ \\ Leesa J. Deterding, ${ }^{3}$ Kenneth A. Jacobson, ${ }^{4}$ and Donald N. Cook ${ }^{1}$ \\ ${ }^{1}$ Immunity, Inflammation and Disease Laboratory, ${ }^{2}$ Integrative Bioinformatics, ${ }^{3}$ Mass Spectrometry Research and Support Group, Epigenetics and Stem Cell Biology Laboratory, National Institute of \\ Environmental Health Sciences, NIH, Research Triangle Park, North Carolina, USA. ${ }^{4}$ Laboratory of Bioorganic Chemistry, National Institute of Diabetes and Digestive and Kidney Diseases, NIH, \\ Bethesda, Maryland, USA.
}

\begin{abstract}
Airway eosinophilia is a hallmark of allergic asthma and is associated with mucus production, airway hyperresponsiveness, and shortness of breath. Although glucocorticoids are widely used to treat asthma, their prolonged use is associated with several side effects. Furthermore, many individuals with eosinophilic asthma are resistant to glucocorticoid treatment, and they have an unmet need for novel therapies. Here, we show that UDP-glucose (UDP-G), a nucleotide sugar, is selectively released into the airways of allergen-sensitized mice upon their subsequent challenge with that same allergen. Mice lacking $P 2 Y_{14} R$, the receptor for UDP-G, had decreased airway eosinophilia and airway hyperresponsiveness compared with wildtype mice in a protease-mediated model of asthma. $\mathrm{P}_{2} \mathrm{Y}_{14} \mathrm{R}$ was dispensable for allergic sensitization and for the production of type 2 cytokines in the lung after challenge. However, UDP-G increased chemokinesis in eosinophils and enhanced their response to the eosinophil chemoattractant, CCL24. In turn, eosinophils triggered the release of UDP-C into the airway, thereby amplifying eosinophilic recruitment. This positive feedback loop was sensitive to therapeutic intervention, as a small molecule antagonist of $\mathrm{P} 2 \mathrm{Y}_{14} \mathrm{R}$ inhibited airway eosinophilia. These findings thus reveal a pathway that can be therapeutically targeted to treat asthma exacerbations and glucocorticoid-resistant forms of this disease.
\end{abstract}

\section{Introduction}

Allergic asthma is a widespread disease characterized by pulmonary inflammation, mucus production, and reversible bronchoconstriction (1). Most, but not all, forms of asthma are driven by type 2 immune responses that can include both $\mathrm{T}$ helper type 2 (Th2) $\mathrm{CD}^{+} \mathrm{T}$ cells and group 2 innate lymphoid cells (ILC2s). Each of these cell types produces type 2 cytokines, including IL-5 and IL-13. IL-5 acts as an eosinophil growth factor in the bone marrow, and in the lung induces chemokines such as eotaxin-2 (CCL24) that can recruit eosinophils to the airway $(2,3)$. IL-13, which is frequently expressed together with IL-5, promotes goblet cell hyperplasia and airway hyperresponsiveness (AHR). In most cases, predominantly eosinophilic forms of allergic asthma respond well to inhaled or oral glucocorticoids, and these drugs have therefore been the mainstay of asthma treatments for many years. However, prolonged steroid use is associated with multiple side effects, including candidiasis, cataracts, and osteoporosis (4). Thus, many patients would benefit from the development of more specific inhibitors of type 2 inflammation that can function as either a stand-alone therapy, or as an adjunctive therapy that

Related Commentary: https://doi.org/10.1172/JCI147735

Conflict of interest: The authors have declared that no conflict of interest exists. Copyright: (5) 2021, American Society for Clinical Investigation.

Submitted: May 29, 2020; Accepted: February 17, 2021; Published: April 1, 2021

Reference information: J Clin Invest. 2021;131(7):e140709.

https://doi.org/10.1172/JCl140709. would allow effective use of lower-dose steroids. Finally, some patients display eosinophilic forms of asthma and other forms of pulmonary diseases that are resistant to glucocorticoids (5-7). Together, these observations underscore the need to develop new drugs that directly target eosinophils.

Several lines of evidence have shown that the initiation and propagation of immune responses are mediated in part by molecules that activate innate immunity (8). Such molecules include endogenous products, sometimes called alarmins, or damageassociated molecular patterns (DAMPs), which alert immune cells to the presence of stressed or dying cells (9). These molecules include proteins, mitochondrial DNA and double-stranded RNA, and nucleotides. ATP is the best-characterized nucleotide alarmin, but recent studies have suggested that other nucleotides and their derivatives, including uridine diphosphate (UDP) and UDP-glucose (UDP-G), might also act as danger signals (10). Among nucleotide ligands, UDP-G and other UDP-sugars bind with highest affinity to $\mathrm{P} 2 \mathrm{Y}_{14} \mathrm{R}$, a $\mathrm{G}$ protein-coupled receptor (GPCR). These ligands are selective for this receptor, whereas at physiological concentrations most other nucleotide agonists of P2YRs typically bind and activate more than one receptor subtype. This exclusive relationship between UDP-sugars and $\mathrm{P}_{2} \mathrm{Y}_{14} \mathrm{R}$ should in principle facilitate studies of how their interaction affects the initiation and progression of immune responses, including those relevant to allergic asthma. Also, UDP-G is not subject to rapid hydrolysis by the same ectonucleotidases such as CD39 that degrade other common P2YR agonists, including nucleoside 5'-triphosphate and $5^{\prime}$-diphosphates (11), and therefore has a longer extracellular 
half-life than ATP (10). Like other nucleotide alarmins, UDP-sugars can be released by stressed cells, including airway epithelial cells (10). Interestingly, UDP-sugars are present at particularly high concentrations in the bronchoalveolar lavage fluid (BALF) of patients with cystic fibrosis (12), suggesting these molecules might promote some forms of pulmonary inflammation.

In the present study, we investigated the role of $\mathrm{P}_{2} \mathrm{Y}_{14} \mathrm{R}$ in a protease-mediated model of asthma that is characterized by robust eosinophilic inflammation and AHR (13). Although we found no evidence that the interaction of UDP-G with $P 2 Y_{14} R$ promotes allergic sensitization through the airway, mice lacking P2 $\mathrm{Y}_{14} \mathrm{R}$ had strongly reduced eosinophilia and AHR following allergen challenge. Similarly, blockade of $\mathrm{P}_{2} \mathrm{Y}_{14} \mathrm{R}$ by a small molecule antagonist during the challenge phase also diminished airway eosinophilia. These results suggest that antagonism of $\mathrm{P} 2 \mathrm{Y}_{14} \mathrm{R}$ might be an effective means to control asthma exacerbations and glucocorticoid-resistant asthma.

\section{Results}

$P 2 Y_{14} R$ is selectively required for pathologic features in proteasemediated models of asthma. Allergic responses to inhaled allergens consist of 2 phases. In the first phase, allergen-specific, naive $\mathrm{CD}^{+} \mathrm{T}$ cells in lung-draining lymph nodes (LNs) differentiate into T helper type 2 (Th2) effector cells and undergo clonal expansion (recently reviewed in ref.14). In the second phase, inhalation of the same allergen triggers recruitment of the allergen-specific effector $\mathrm{CD} 4^{+} \mathrm{T}$ cells to the lung, their release of signature cytokines, and consequent airway inflammation. Previous work has shown that administration of a highly purified protein such as ovalbumin (OVA) into the airway leads to immunotolerance, whereas including an adjuvant with that protein overcomes tolerance and drives adaptive immunity (15). Toll-like receptor (TLR) ligands and proteases can each function as adjuvants to promote allergic sensitization through the airway, although these 2 classes of compounds act through different innate immune signaling pathways and give rise to distinct forms of allergic airway disease (13). To determine if the interaction of UDP-sugars with $\mathrm{P} 2 \mathrm{Y}_{14} \mathrm{R}$ contributes to disease features in either of these general models of asthma, we sensitized wild-type C57BL/6J mice and genetically matched $\mathrm{P} 2 \mathrm{ry} 14^{-/-}$mice by airway administrations of the test allergen OVA alone, or OVA mixed together with LPS, the cysteine protease papain (PAP), or a protease complex prepared from Aspergillus oryzae (ASP) (13). One week after the second of 2 such sensitizations, the animals were challenged by exposure to an aerosolized solution of OVA without adjuvant (Figure 1A). As expected from previous studies $(13,16)$, wild-type mice sensitized to OVA alone did not develop inflammation upon subsequent exposure to the allergen (Figure 1B). By contrast, when LPS was used as an adjuvant, the mice developed robust neutrophilia, along with modest eosinophilia. Animals sensitized using either PAP or ASP as the adjuvant developed strong eosinophilia, but relatively weak neutrophilia. Compared with wild-type animals, $\mathrm{P} 2 \mathrm{ry} 14^{-/-}$mice had markedly and significantly reduced eosinophilia in both the ASP/OVA and PAP/OVA models of asthma. No genotype-specific differences in eosinophils were seen when animals were sensitized through the peritoneum, using aluminum hydroxide (alum) as the adjuvant (Supplemental Figure 1; supplemental material available online with this article;
https://doi.org/10.1172/JCI140709DS1). Together, these findings demonstrate that $\mathrm{P} 2 \mathrm{Y}_{14} \mathrm{R}$ selectively promotes airway eosinophilia in protease-mediated models of asthma.

The shortness of breath (dyspnea) experienced by asthmatics stems in part from AHR. To determine whether signaling through $\mathrm{P} 2 \mathrm{Y}_{14} \mathrm{R}$ contributes to this physiologic response, we again sensitized wild-type and P2ry $14^{-/-}$mice with either ASP/OVA or LPS/ OVA, and challenged them with aerosolized OVA. Invasive measurements of airway resistance were performed in anesthetized mice following airway administrations of various doses of the muscarinic receptor agonist methacholine. As expected from previous work (13), wild-type mice undergoing the ASP/OVA model of asthma developed stronger AHR than did mice in the LPS/OVA model (Figure 1C). P2 $\mathrm{Y}_{14} \mathrm{R}$ was required for this response because compared with wild-type mice, $P 2 r y 14^{-/-}$mice had markedly attenuated AHR in the ASP/OVA model of asthma. No genotype-specific differences were noted in the LPS/OVA model.

IL-5 is a well-described growth factor for eosinophils, and together with eosinophil chemoattractants promotes eosinophil recruitment to the lung (3). However, despite their pronounced differences in degree of eosinophilic inflammation, airways of wild-type and P2ry14 $14^{-/}$mice had similar amounts of IL-5 (Figure 1D). IL-13 was also present at similar amounts in these 2 strains. In agreement with these observations, there were also no differences between wild-type and $\mathrm{P} 2 \mathrm{ry} 14^{-/-}$mice in serum concentrations of OVA-specific IgE (Figure 1E) or in mucus-producing cells (Figure $1 \mathrm{~F})$. Together, these data show that in protease-mediated models of asthma, $\mathrm{P}_{2} \mathrm{Y}_{14} \mathrm{R}$ has an important role in the development of eosinophilic inflammation and AHR, but is dispensable for other features associated with type 2 asthma.

$P 2 Y_{14} R$ is dispensable for allergic sensitization through the airway. The reduced airway inflammation and AHR seen in P2ry $14^{-/-}$animals might have been due to a role of $\mathrm{P} 2 \mathrm{Y}_{14} \mathrm{R}$ during the sensitization phase, or to a requirement of that receptor during the challenge phase. Although instillation of UDP-G into the airways of naive mice promoted neutrophilia, it was likely due to contaminating endotoxin, as this response was also seen in $P 2 r y 14^{-/-}$recipient mice, but not in $\mathrm{Tlr}^{-/-}$mice (Supplemental Figure 2). To circumvent this issue, we studied allergic sensitization using $\mathrm{P} 2 \mathrm{ry} 14^{-/-}$recipient mice. We adoptively transferred naive, OVA-specific $\mathrm{CD} 4^{+} \mathrm{T}$ cells from OT-II mice into either wild-type or $\mathrm{P} 2 \mathrm{ry} 14^{-/-}$recipient mice and sensitized the animals with ASP/OVA. Four days later, lung-draining mediastinal LNs (mLNs) were excised and cells from them were cultured ex vivo in the presence of exogenous OVA (Figure 2A). Analysis of cytokines in the $\mathrm{mLN}$ culture supernatants did not reveal genotype-specific differences in IL-5 or IL-13, and as expected for this protease model of asthma, very little IL-17 was produced (Figure 2B). To confirm that $\mathrm{P} 2 \mathrm{Y}_{14} \mathrm{R}$ is dispensable during the sensitization phase of the protease-dependent model of asthma, we employed the $\mathrm{P} 2 \mathrm{Y}_{14} \mathrm{R}$ antagonist, 4-[4-(piperidin-4-yl)phenyl]-7-[4-(trifluoromethyl)phenyl]naphthalene-2-carboxylic acid (PPTN), during allergic sensitization (Figure 2C). Following subsequent OVA challenge, animals that had received PPTN during sensitization responded similarly to vehicle-treated mice (Figure 2D). Together, these data indicate that $\mathrm{P} 2 \mathrm{Y}_{14} \mathrm{R}$ is dispensable for allergic sensitization and suggest that this receptor might instead be required during the challenge phase of the model. 
A

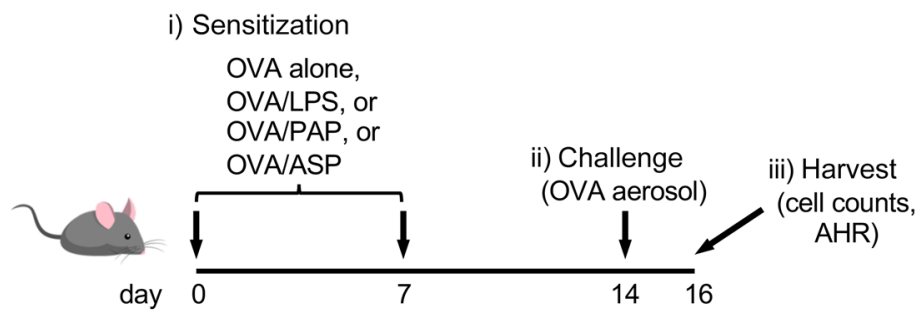

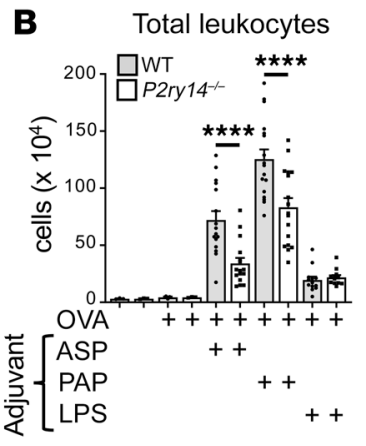

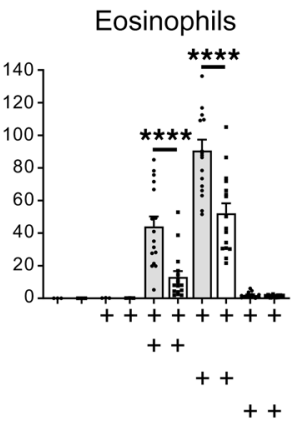

C
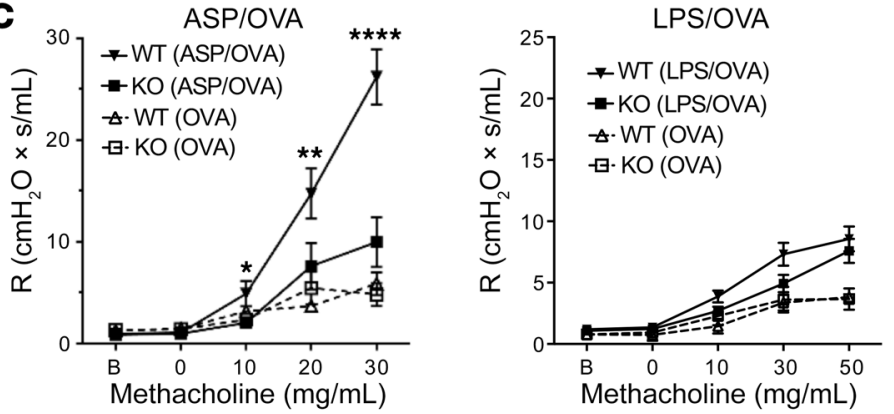

\section{E}

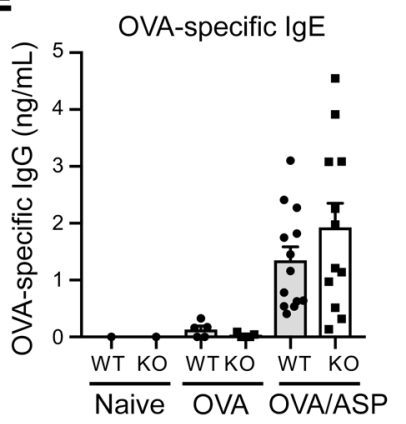

F OVA only

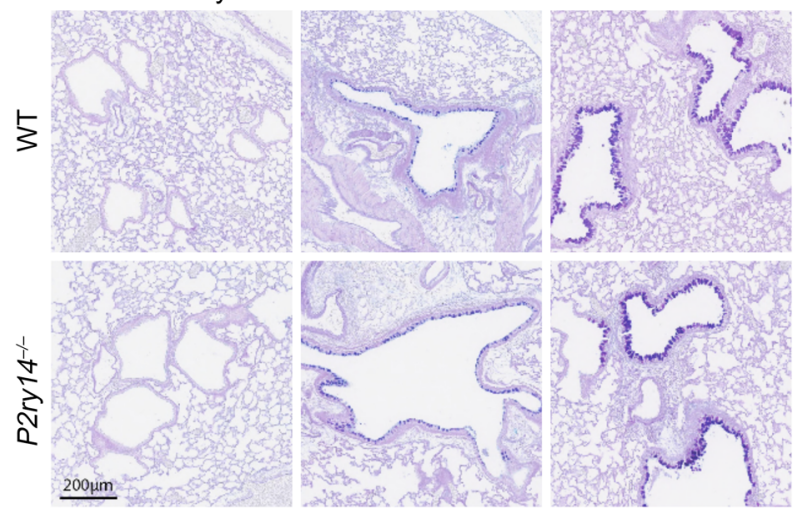

D
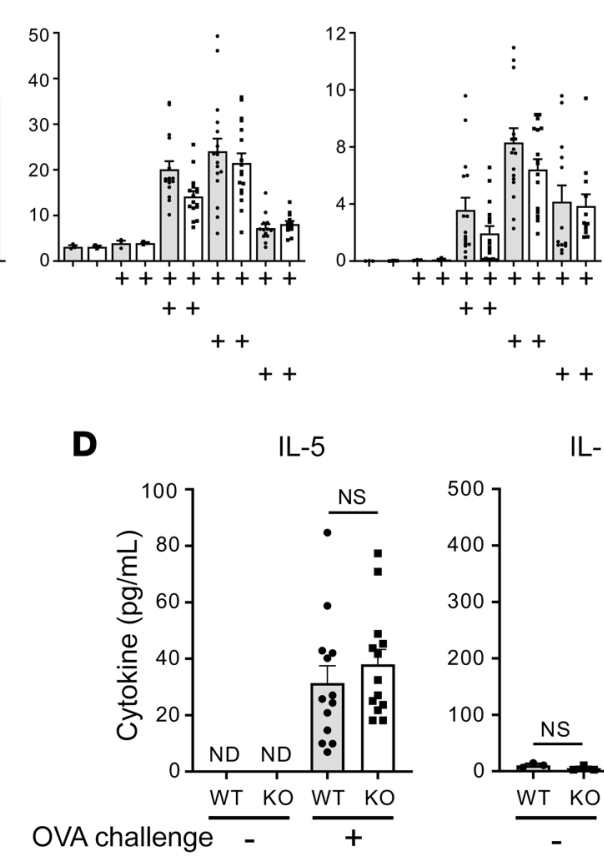

Lymphocytes

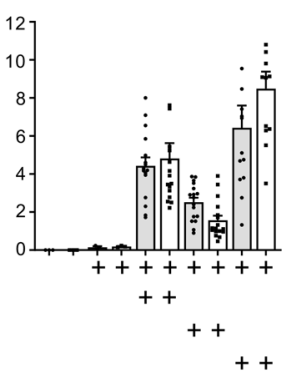

IL-13

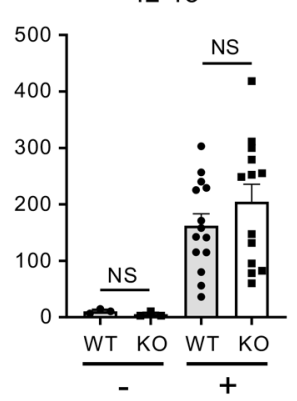

Mucin-staining cells

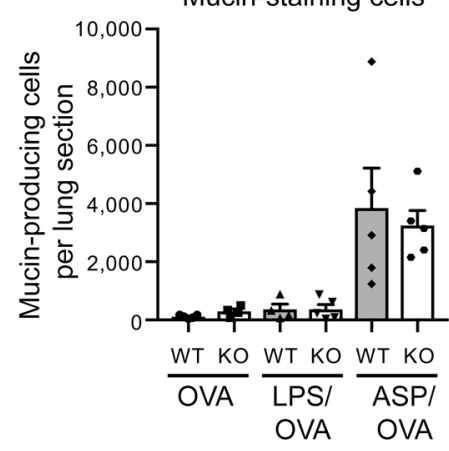

Figure 1. P2ry14 is required for airway eosinophilia and AHR in protease-mediated models of asthma. (A) Timeline showing allergic sensitization, challenge, and harvest of C57BL/6) wild-type and genetically matched P2ry14 ${ }^{-/}$(KO) mice in LPS- and protease-mediated (ASP and PAP) models of asthma. (B) Airway inflammation at 48 hours after allergen challenge in wild-type mice (gray rectangles) and $P 2$ ry $14^{4^{-/}}$mice (white rectangles) previously sensitized using the indicated adjuvant. Shown are mean values \pm SEM $(n=12-17$ per group, except for nontreated or OVA-only-treated groups, where $n=3)$. (C) Airway resistance (R) measurements in OVA-challenged mice previously sensitized using OVA only, ASP/OVA, or LPS/OVA. Mean ( \pm SEM) values are shown $(n=10)$ for baseline (B) and after administration of the indicated doses of methacholine. Results shown are from a single experiment, representative of 2 . ND, not detectable. ${ }^{*} P<0.05$; ${ }^{* *} P<0.01$; ${ }^{* * *} P<0.0001$; NS, not significant; analyzed using 1-way ANOVA followed by Bonferroni's post hoc test. (D) Concentrations of IL-5 and IL-13 in BALF after OVA challenge in ASP/OVA asthma model. (E) Concentrations of OVA-specific IgE in serum. Means \pm SEM are shown for naive mice, and for OVA with and without ASP-sensitized and OVA-challenged animals. (F) Representative Alcian blue- and periodic acid-Schiff-stained sections showing mucus-producing cells. Sections were prepared from lungs of [57BL/6] wild-type and genetically matched P2ry14 ${ }^{--}$mice (left). Scale bar: $200 \mu \mathrm{m}$. Also shown are compiled data for mean numbers \pm SEM of mucus-producing cells in individual lung slices from the indicated groups. 
A

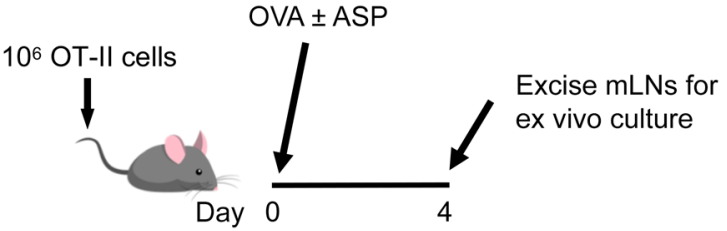

B
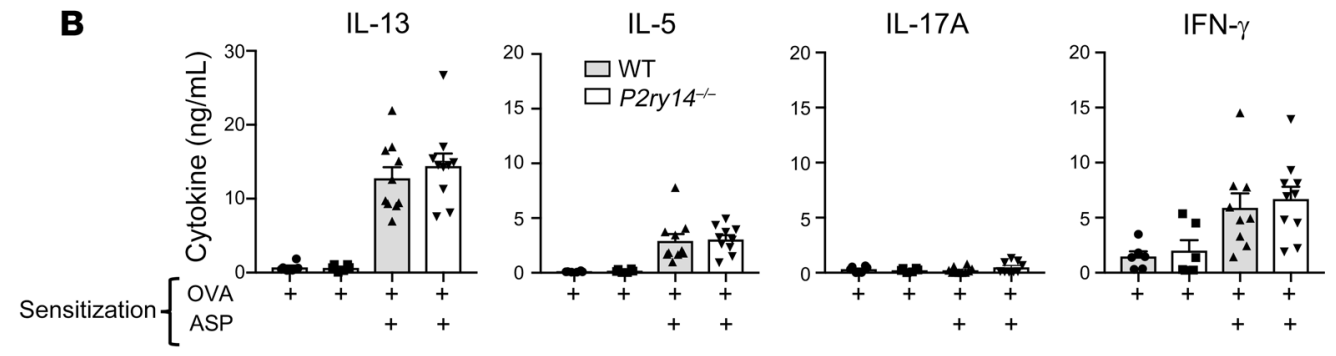

Figure 2. P2ry14 is dispensable for allergic sensitization through the airway. (A) Timeline for injection of OVA-specific (OT-II) T cells into WT or P2ry14-deficient (KO) mice, followed by ASP/OVA sensitization and harvest of mLNs. (B) Concentrations of the indicated cytokines in culture supernatants of $\mathrm{mLNs}$ harvested from mice treated with OVA alone or OVA/ASP. (C) Timeline for PPTN treatments, 2 OVA/ASP sensitizations, and a single OVA aerosol challenge. (D) Effect of PPTN treatments during allergic sensitization on cell numbers of the indicated leukocyte subsets following OVA challenge. Data shown represent mean values \pm SEM.

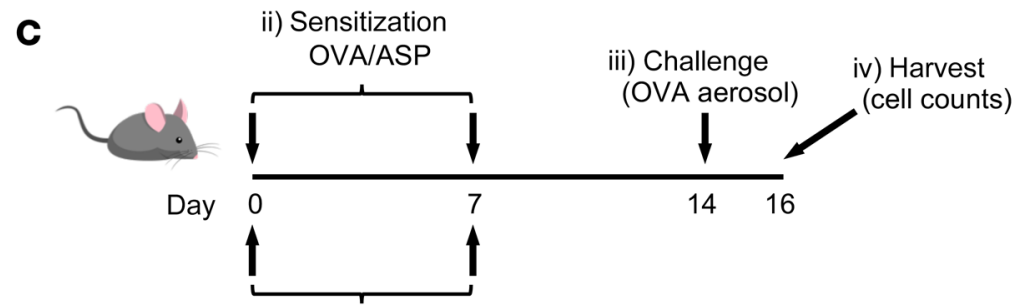

i) i.p. injection
PPTN $(10 \mathrm{mg} / \mathrm{kg})$,
or vehicle

D

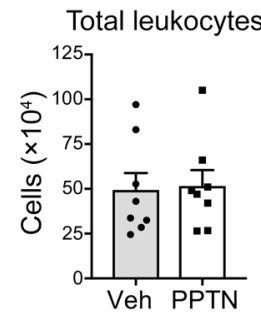

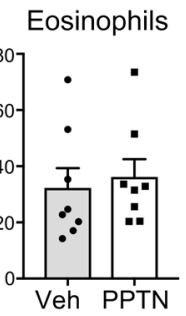

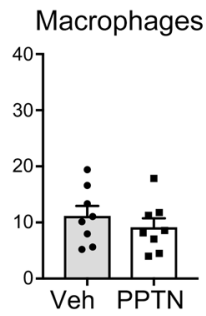

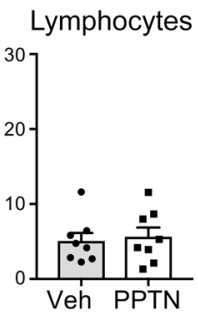

$P 2 Y_{14} R$ promotes eosinophilic inflammation during allergen challenge. To directly test the role of $\mathrm{P}_{2} \mathrm{Y}_{14} \mathrm{R}$ during allergen challenge, we sensitized wild-type mice with ASP/OVA to allow allergen-specific $\mathrm{T}$ cells to develop normally, but then blocked $\mathrm{P} 2 \mathrm{Y}_{14} \mathrm{R}$ function during the challenge phase by administration of PPTN (Figure 3A). This treatment reduced the number of total cells in the airways of the challenged mice (Figure 3B). Differential analysis of airway leukocytes revealed that this decrease was primarily due to reductions in eosinophil recruitment. Lymphocytes were also reduced by the PPTN treatment, but macrophages were not. The ability of small molecule antagonists of $\mathrm{P} 2 \mathrm{Y}_{14} \mathrm{R}$ to reduce eosinophilia in this mouse model suggests that a similar approach might be an effective means to reduce or prevent eosinophilia in human asthmatics.

$P 2 Y_{14} R$ functions in leukocytes to promote airway eosinophilia. Having found that $\mathrm{P}_{2} \mathrm{Y}_{14} \mathrm{R}$ promotes eosinophilic accumulation in the airway during the challenge phase, we next sought to identify the cell type(s) in which P2ry14 expression is required to sustain robust eosinophilic inflammation. We first determined whether $\mathrm{P} 2 \mathrm{Y}_{14} \mathrm{R}$ is functioning primarily in structural cells or hematopoi- etic cells by generating reciprocal bone marrow-chimeric mice using wild-type and $P 2 r y 14^{-/}$animals. After allowing 10 weeks for recipient mice to be fully reconstituted, they were sensitized using ASP/OVA and subsequently challenged with aerosolized OVA. As expected, irradiated wild-type animals that received wild-type bone marrow cells developed robust airway eosinophilia (Figure 4A). Similar numbers of eosinophils were seen in airways of $P 2 r y 14^{-/}$recipient mice that had received wild-type donor marrow, indicating that $\mathrm{P}_{2} \mathrm{Y}_{14} \mathrm{R}$ in radioresistant cells is largely dispensable for eosinophilic inflammation. By contrast, wild-type mice or P2ry14 ${ }^{-/}$animals that received P2ry $14^{-/-}$bone marrow had significantly reduced eosinophilia compared with the other 2 groups. These findings demonstrate that $\mathrm{P} 2 \mathrm{Y}_{14} \mathrm{R}$ in hematopoietic cells is required for eosinophilic inflammation in the proteasemediated model of asthma.

P2ry14 is most highly expressed in lung eosinophils. To gain a better understanding of which hematopoietic cells might be important for eosinophilic inflammation, we used flow cytometry to isolate 8 different types of lung leukocytes, along with 2 different types of structural cells: endothelial cells and epithelial cells. 
A

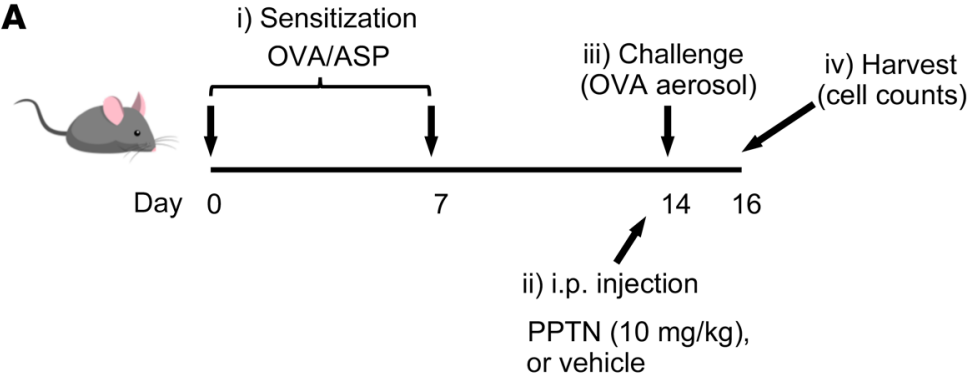

Figure 3. $\mathbf{P} 2 Y_{14} \mathbf{R}$ blockade during allergen challenge diminishes eosinophilic airway inflammation. (A) Timeline for ASP/OVA sensitization, PPTN treatment, and OVA challenge. (B) Effect of PPTN administration during challenge on airway inflammation in OVAchallenged mice previously sensitized with ASP/OVA. Data shown represent mean values \pm SEM. ${ }^{*} P<0.01$; ${ }^{* *} P=0.0003$ by 2 -tailed Student's $t$ test.
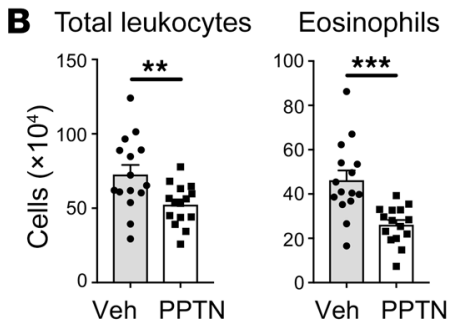

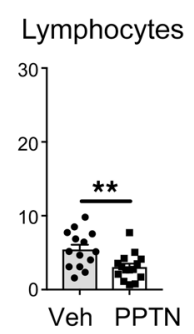

Veh PPTN Veh PPTN

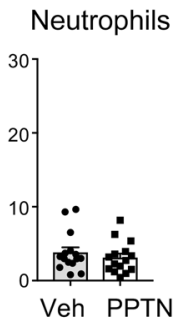

To further study the role of P2ry14 in individual leukocyte subsets that express this gene, we crossed conditionally mutant, $P 2 r y 14^{f / f l}$ animals to mice bearing different $\mathrm{Cre}$ recombinase transgenes. As expected, $P 2 r y 14^{f / / l}$ mice (having no Cre transgene) responded similarly to wild-type C57BL/6J animals in the ASP/ OVA model of asthma, whereas $P 2 r y 14^{-/}$mice had reduced eosinophilic inflammation. LysM-Cre-mediated deletion of P2ry14 in myeloid cells, such as macrophages and neutrophils, but not eosinophils (ref. 17, P2ry14 $4^{\text {Imyeloid }}$ mice), did not reduce eosinophilia (Figure $4 \mathrm{~F}$ and Supplemental Figure 3), nor did Cd11c-Cre-mediated deletion of P2ry14 in antigen-presenting cells (APCs) (P2ry14 ${ }^{\triangle A P C}$ mice) (18). However, selective deletion of P2ry14 in eosinophils through expression of Cre at the eosinophil peroxidase locus (19) (P2ry14 ${ }^{\text {seos }}$ mice) markedly reduced airway eosinophilia (Figure 4G). Together, these data show that P2ry14 expression in neutrophils, macrophages, and other APCs is dispensable for eosinophilic inflammation, whereas expression of this gene in eosinophils themselves is important for their recruitment to the airway.

Allergic challenge triggers release of UDP-hexoses. Our results thus far suggested that UDP-G is released into the airway during the challenge phase, and that this release promotes eosinophil migration. To test this, we used mass spectrometry to measure total UDP-hexoses in BALF of mice, as this procedure cannot distinguish between UDP-G and other UDP-hexoses. Lungs of euthanized mice were lavaged with PBS previously spiked with ${ }^{13} \mathrm{C}_{6}$-UDP- $\mathrm{G}$, which served as an internal standard to which endogenous UDP-hexoses were normalized to control for variability in recovery from lavage fluid (Supplemental Figure 4). We found that although endogenous UDP-hexoses were present in the airway at steady state, these levels were significantly increased after OVA challenge of mice previously sensitized using ASP/OVA (Figure 5A). UDP-hexoses did not increase following OVA challenge of mice sensitized using OVA only, nor after OVA challenge of mice previously sensitized with LPS/OVA, which display neutrophilic inflammation, but not prominent eosinophilia (Figure 5A). Thus, production of UDP-hexoses was only increased when significant eosinophilia was already present in the airway. Interestingly, peak $\mathrm{P} 2 \mathrm{Y}_{14} \mathrm{R}$ likely acts in an eosinophil-int
recruitment of those cells to the airway. 
A

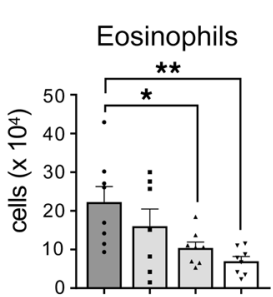

Donor P2ry14 +/+ +/+ - $1--1-$

Recipient P2ry14 +/+ -/- +/+ - /-

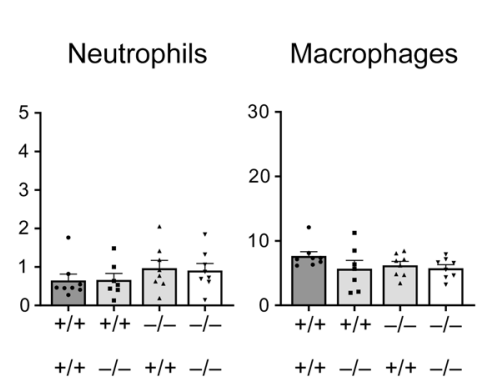

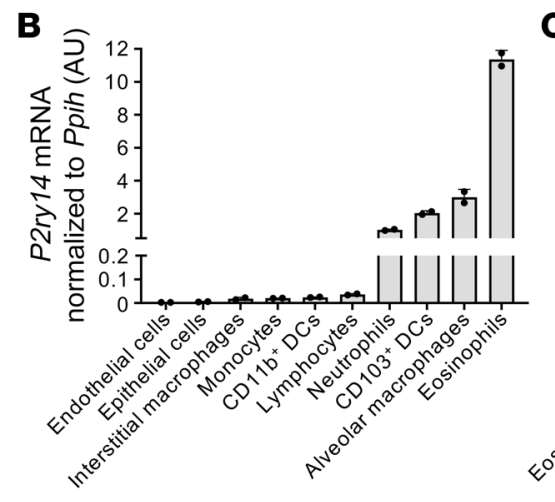

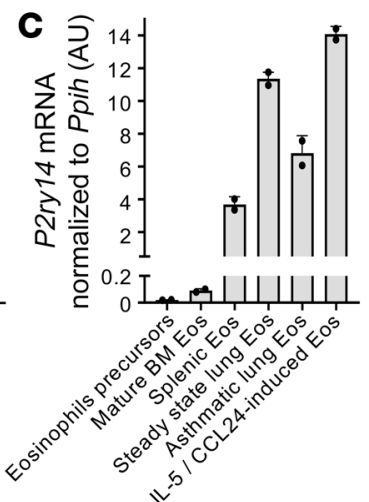

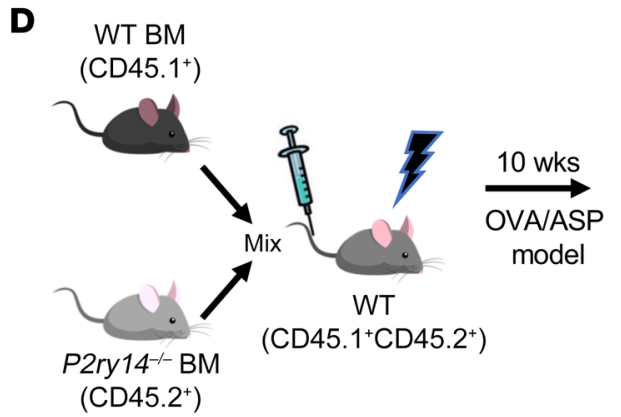
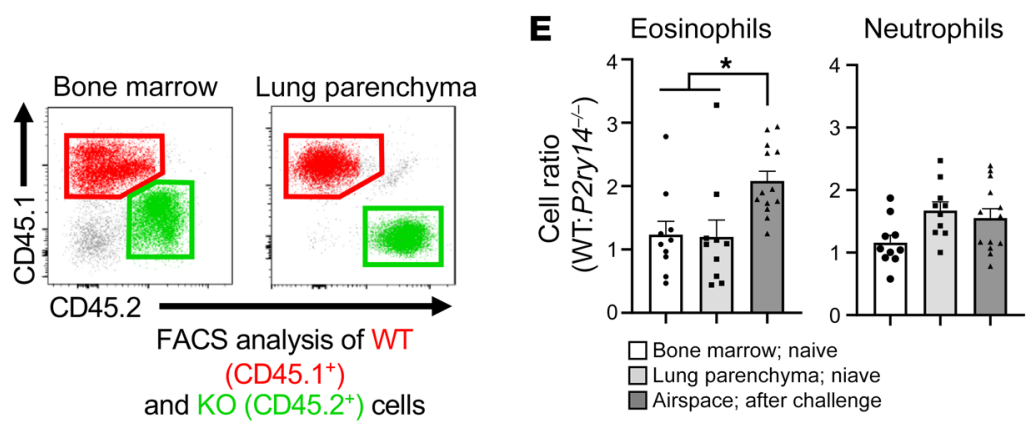

$\mathbf{F}$ LyzM-cre
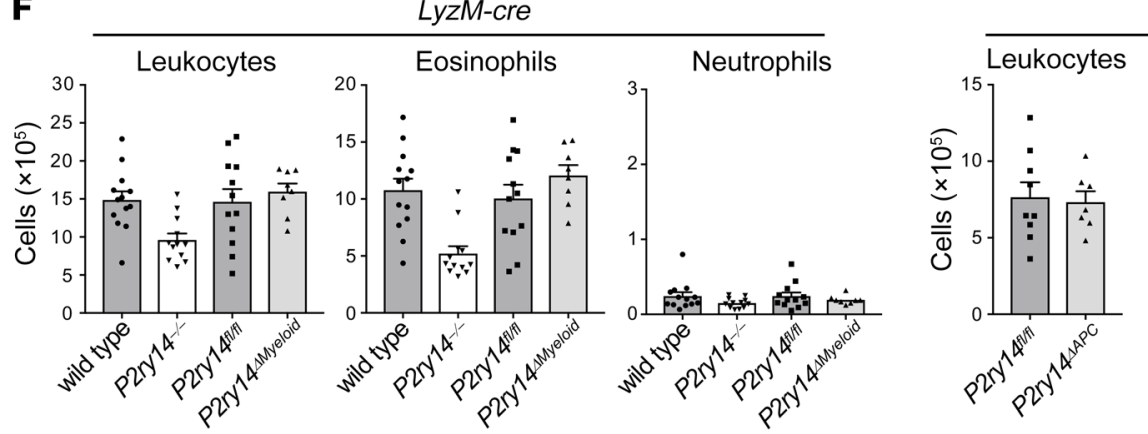

Cd11c-cre

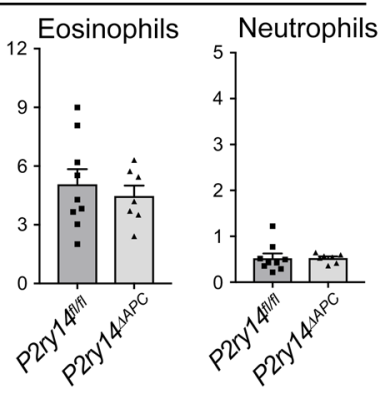

G

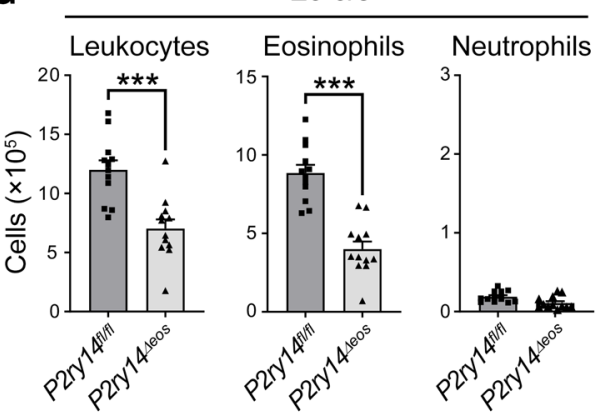

Figure 4. Expression of P2ry14 in lung eosinophils is essential for eosinophilic airway inflammation. (A) Cell numbers for the indicated leukocyte types in airways of reciprocal bone marrow-chimeric (BM-chimeric) mice generated using WT and P2ry $14^{-/-}$animals and subjected to the ASP/OVA model of asthma. (B and C) Expression of P2ry14 in the indicated populations of leukocytes and stromal cells from lungs of C57BL/6) mice (B), and in eosinophils during their maturation and migration to the periphery (C). (D) Generation of mixed BM chimeras by injecting equal numbers of BM cells from WT (CD45.1 $\left.1^{+}\right)$ and P2ry14-null (CD45.2+) mice into irradiated Cd45.1/Cd45.2 heterozygous recipients. Dot-plot cytograms show representative flow cytometry results for CD45.1 and CD45.2 staining of BM (left) and lung cells (right) of nonchallenged mice, indicating similar reconstitution of leukocyte populations of each

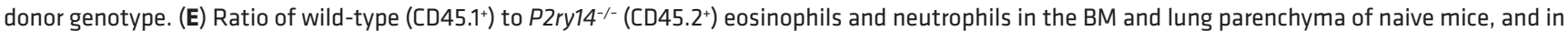
the airspace of OVA-challenged mice previously sensitized with ASP/OVA. (F) Allergic airway inflammation in mice with conditional P2ry14 disruption in LyzM-expressing myeloid cells (left), and disruption in Cd11c-expressing alveolar macrophages and dendritic cells (right). (C) Allergic airway inflammation in mice with $P 2$ ry14 disruption in eosinophils. Data shown represent mean values $\pm \mathrm{SEM}$. ${ }^{*} P<0.05 ;{ }^{* *} P<0.01 ;{ }^{* *} P<0.001$. Data were analyzed using 2-tailed Student's $t$ test for pairwise comparisons, or 1-way ANOVA followed by Tukey's post hoc test for multiple comparisons. 
production of UDP-sugars in the airway preceded peak accumulation of eosinophils (Figure 5, B and C), suggesting that eosinophils might trigger UDP-G release that in turn promotes more eosinophilia in a positive feedback loop.

UDP-sugars act through $P 2 Y_{14} R$ to promote responsiveness to eosinophil chemoattractants. Qualitative analysis of precision-cut lung slices (PCLS) indicated that eosinophils were more closely associated with $\mathrm{CD}^{2} 1^{+}$endothelial cells in lungs of $\mathrm{P} 2 \mathrm{ry} 14^{-/-}$mice than in lungs of wild-type mice (Supplemental Figure 5). This suggested that eosinophils lacking the $\mathrm{P} 2 \mathrm{Y}_{14} \mathrm{R}$ axis might move less efficiently from the vascular bed to the lung interstitium and airway. To confirm this, we labeled marginated eosinophils by i.v. injections of anti-CD45 antibodies 5 minutes prior to euthanasia and perfusion of the lungs. Flow cytometric analysis of the lungs revealed that there were indeed more marginated and blood eosinophils in P2ry14 ${ }^{-/-}$mice than in wild-type mice (Figure 5D). One possible explanation for this was that UDP-G, acting through $\mathrm{P} 2 \mathrm{Y}_{14} \mathrm{R}$, indirectly recruited eosinophils by increasing production of eosinophil chemoattractants. However, neither of the eosinophil-attracting chemokines, CCL11 and CCL24, were reduced in airways of $\mathrm{P} 2 \mathrm{ry} 14^{-/-}$mice compared to those in wild-type mice (Figure 5E). Indeed, CCL24 was significantly higher in P2ry $14^{-/}$ animals than in their wild-type counterparts. RNA sequencing (RNA-seq) of wild-type and P2ry14 ${ }^{-/-}$eosinophils did not reveal major differences in their transcriptomes (Supplemental Figure 6, A and B). Although some genotype-specific differences were noted in genes governing cell adhesion, the magnitude of these differences was very small (Supplemental Figure 6C). Mean fluorescence intensity (MFI) levels for CCR3, the receptor for CCL11 and CCL24, were also similar on eosinophils prepared from the lungs, bone marrow, and blood of wild-type and $\mathrm{P} 2 \mathrm{ry} 14^{-/-}$mice (Figure $5 \mathrm{~F}$ and Supplemental Figure 7A). No genotype-specific differences were noted in either apoptotic or nonapoptotic cell death in blood and airway eosinophils, as determined by flow cytometry experiments in which annexin $\mathrm{V}$ was used to evaluate apoptosis and a cell viability dye (EF78) to detect necrosis (Supplemental Figure $7 \mathrm{~B}$ ), and there were no genotype-specific differences in the display of the IL-5 receptor, IL5R $\alpha$ (CD125) (Supplemental Figure 7C).

We next investigated whether UDP-sugars act directly on eosinophils, or alternatively, increase their responsiveness to other chemoattractants. To do this, we harvested eosinophils from lungs of ASP/OVA-sensitized and OVA-challenged wild-type and P2ry $14^{-/-}$mice, and placed the cells in the top well of a Boyden chamber. Cell migration was assessed by analysis of cells that moved from the top to the bottom chamber (Figure $5 \mathrm{G}$ ). Adding UDP-G to the bottom chamber promoted migration of wild-type eosinophils (Figure 5H). However, enhanced migration was also seen when UDP-G was added to both the top and bottom chambers, suggesting that UDP-sugars enhance chemokinesis (nondirectional cell movement), as opposed to directional chemotaxis. This activity was not due to contaminating endotoxin, as eosinophils from Tlr4-deficient mice also responded similarly to UDP-G (Supplemental Figure 8A). The activity was specific for $P 2 \mathrm{Y}_{14} \mathrm{R}$, however, as it was not seen with eosinophils from $\mathrm{P} 2 \mathrm{ry} 14^{-/-}$mice (Figure $5 \mathrm{H}$ ). As expected, adding the eosinophil chemoattractant CCL24 to the bottom chamber also enhanced the migration of eosinophils from the top to the bottom chamber. The presence of UDP-G in the top and bottom chambers further increased the CCL24-mediated chemotaxis, and again, this activity was dependent on $\mathrm{P} 2 \mathrm{Y}_{14} \mathrm{R}$, as it was not seen in eosinophils from $\mathrm{P} 2 \mathrm{ry} 14^{-/-}$ mice (Figure $5 \mathrm{H}$ ), nor with immature eosinophils from the bone marrow, which lack appreciable P2ry14 expression (Supplemental Figure $8 \mathrm{~B}$ ). Further, this activity could be blocked by the $\mathrm{P} 2 \mathrm{Y}_{14} \mathrm{R}$ inhibitor PPTN (Supplemental Figure 8C). Together, these data show that UDP-sugars increase the chemokinetic activity of eosinophils in vitro, thereby enhancing the ability of eosinophil-attracting chemokines to direct eosinophil chemotaxis. In vivo, instillation of UDP-G into the airways of ASP/OVA-sensitized mice following OVA challenge further increased eosinophilia of the airway (Supplemental Figure 9).

Eosinophilic inflammation promotes release of UDP-sugars into the airway. We next investigated potential mechanisms to explain our finding that UDP-G is increased after OVA challenge of mice previously sensitized using ASP/OVA, but not in similarly challenged mice that had been previously sensitized with either LPS/OVA or OVA alone (Figure 5A). Noting that of these 3 groups, mice sensitized using ASP/OVA also had the most eosinophils after challenge (Figure 1B), we considered the possibility that eosinophils themselves might trigger production of UDP-G. To test that hypothesis, we prepared eosinophils from airways of mice carrying 1 transgene for IL-5 and another for lung expression of CCL24. These doubletransgenic mice (hE2 $\times$ NJ.1638 mice) display spontaneous pulmonary eosinophilia. In parallel, we prepared neutrophils from airways of CXCL1/CXL5-treated C57BL/6J mice. These 2 cell types were separately transferred into the airways of naive mice, and their lungs subjected to BAL 4 hours later for analysis of UDP-hexoses by mass spectrometry. Mice that received adoptive transfer of eosinophils displayed a significant increase in UDP-hexoses, compared with mice that received no cells (Figure 5I). Adoptive transfer of neutrophils, by contrast, had no effect on airway levels of UDP-G.

To confirm a role for eosinophils in the production of UDP-G, we treated mice prior to challenge with an antibody against CCR3. This treatment only partially reduced numbers of lung eosinophils in mice undergoing the asthma model (Figure 5J), likely because bone marrow eosinophils, which lack CCR3 and are not efficiently killed by antibodies against this protein (Supplemental Figure 10), can migrate to the lung following allergen challenge. Nonetheless, reducing eosinophils by this method led to comparable reductions in UDP-G compared with mice that did not receive depleting antibodies (Figure 5K). Together, these data indicate that eosinophils trigger release of UDP-G, which in turn promotes the recruitment of more eosinophils by augmenting chemokinesis and CCL24-driven chemotaxis.

\section{Discussion}

Our current findings reveal that UDP-G signaling through $\mathrm{P}_{2} \mathrm{Y}_{14} \mathrm{R}$ has a pivotal role in an animal model of allergic asthma. Although dispensable for both eosinophil and neutrophil recruitment to the airway in a TLR ligand-promoted model of asthma, P2ry14 was required for eosinophil recruitment in a proteasemediated model of that disease. The ability of a $\mathrm{P} 2 \mathrm{Y}_{14} \mathrm{R}$ antagonist to similarly suppress allergen-induced eosinophilia suggests that targeting the UDP-G/P2 $\mathrm{Y}_{14} \mathrm{R}$ axis might be an effective means to treat eosinophilic asthma. 
A

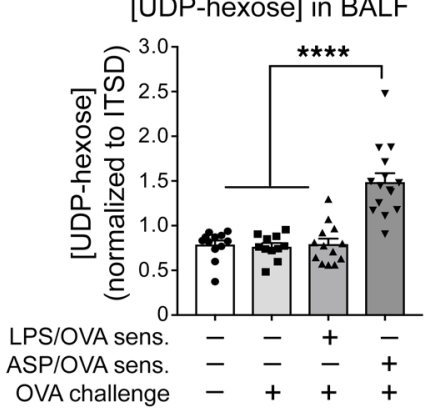

B

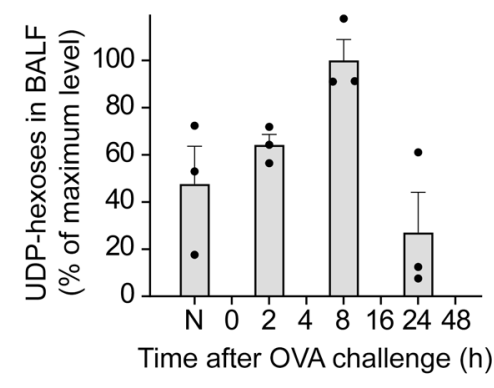

C

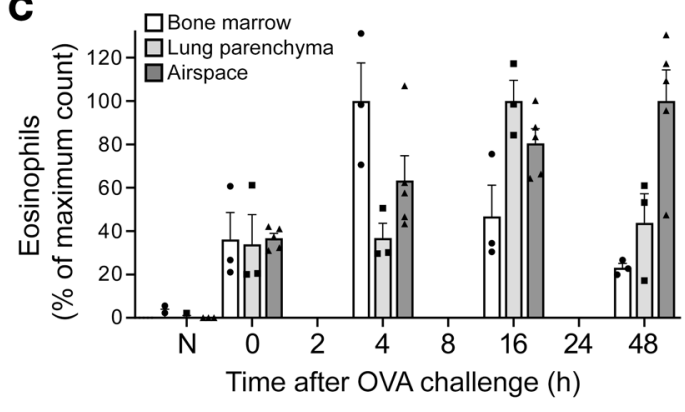

D

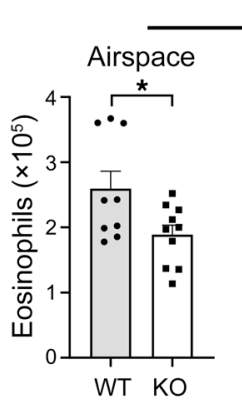

G
Eosinophils

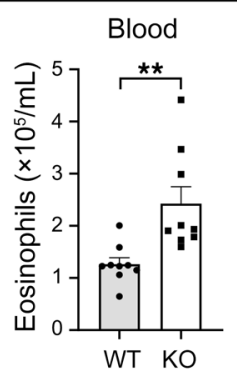

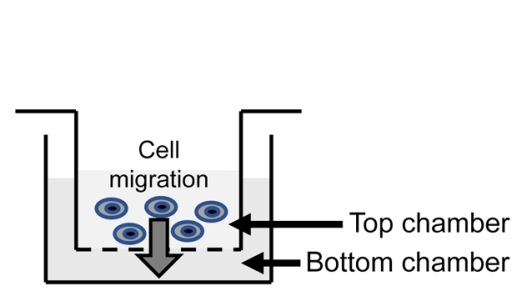

H

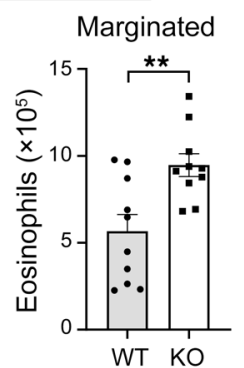

WT KO
E

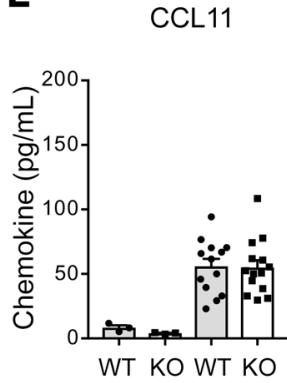

CCL24

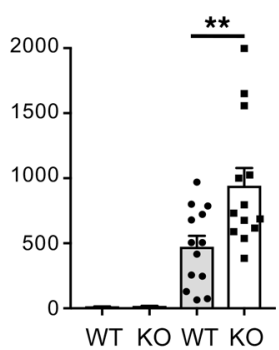

F

CCR 3

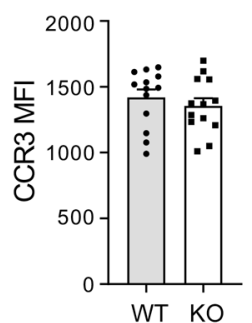

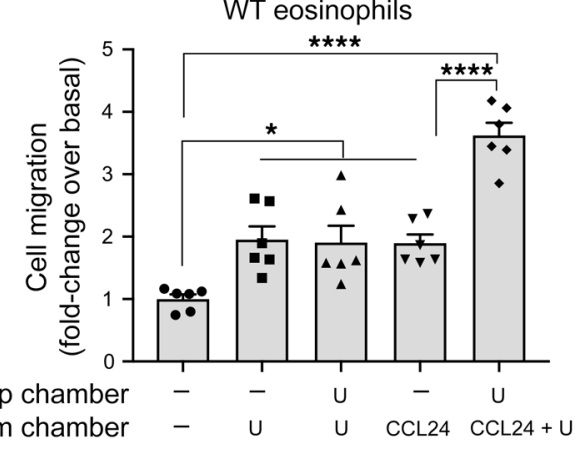
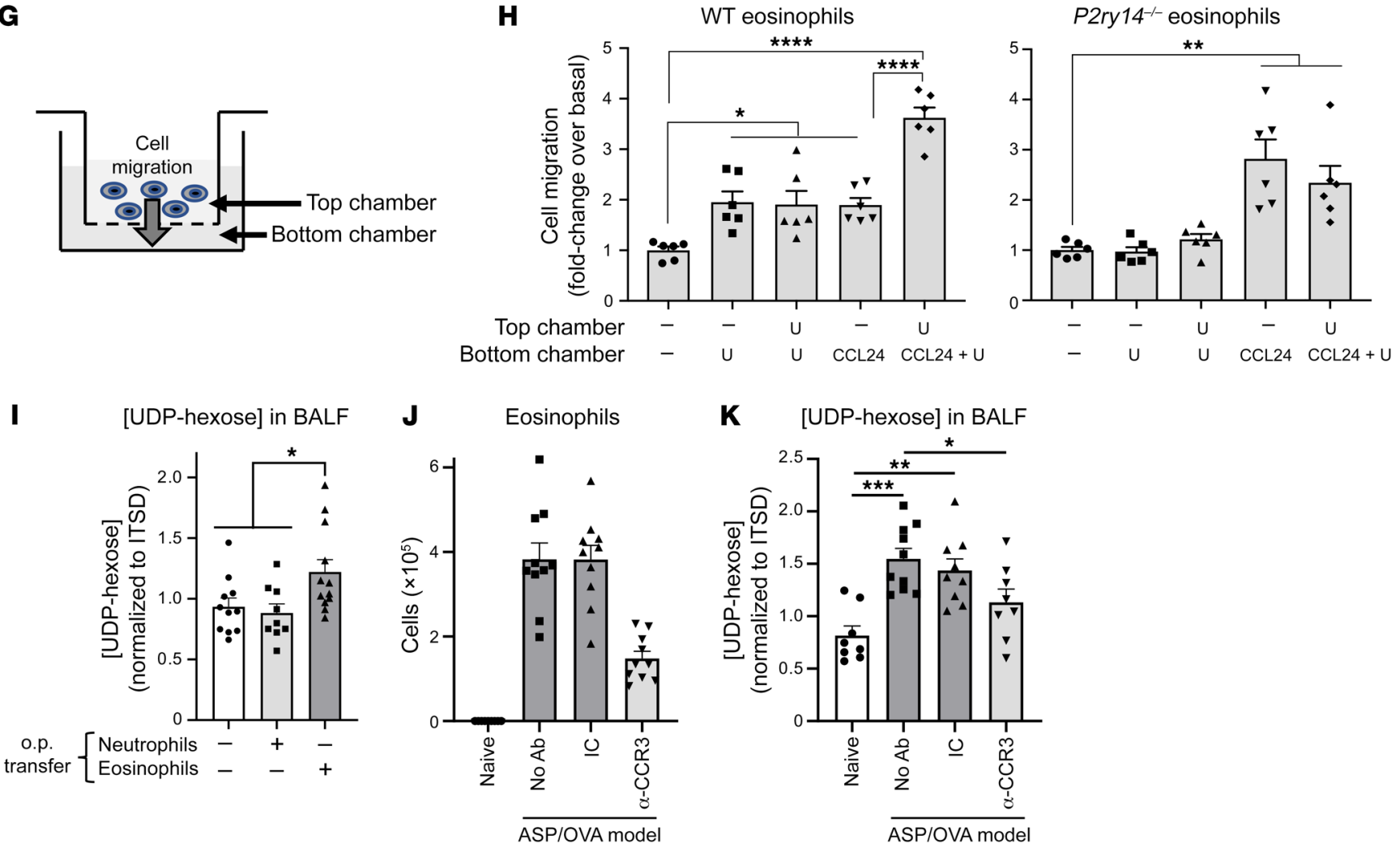

$\mathbf{K}$ [UDP-hexose] in BALF

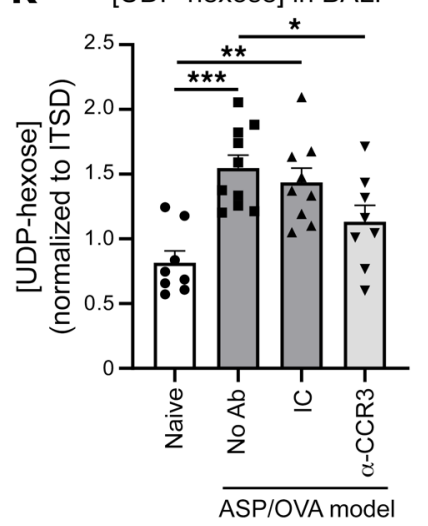

Figure 5. UDP-glucose amplifies eosinophil recruitment to the airway. (A) Mass spectrometry measurements of UDP-hexoses in BALF 8 hours after OVA challenge of mice previously sensitized using ASP/OVA or LPS/OVA. Data represent sample readouts relative to the internal standard (ITSD), ${ }^{13} \mathrm{C}_{6}-\mathrm{UDP}$ glucose (included in the fluid used for lavage). (B and C) Time course for airway levels of UDP-hexoses (B), and for eosinophil numbers in the indicated locations (C). Analyses were performed on [57BL/6] mice sensitized twice with OVA/ASP, challenged once with OVA, and harvested at the indicated times. Data shown represent means \pm SEM $(n=3-5)$ from a single experiment. (D) Air space, blood, and marginated eosinophils in ASP/OVA-sensitized mice at 16 hours after OVA challenge. (E) Airway levels of CCL11 and CCL24 in ASP/OVA-sensitized mice, 4 hours after OVA challenge. (F) MFI of CCR3 on lung eosinophils. (C) Schematic representation of in vitro cell migration assay. (H) Migration of WT and P2ry14 ${ }^{-/-}$eosinophils in response to the indicated agents added to the top and/or bottom chambers. U, UDP-glucose. (I) UDP-hexose levels in BALF after adoptive transfer of the indicated cells into the airways. (J) Effect of anti-CCR3 antibody on lung eosinophils. (K) Effect of anti-CCR3 antibody on UDP-hexoses in BALF. IC, isotype control antibody. Data shown represent mean values \pm SEM. ${ }^{*} P<0.05 ;{ }^{* *} P<0.01 ;{ }^{* * *} P<0.001 ;{ }^{* * *} P<0.001$. Data were analyzed using 2-tailed Student's $t$ test for pairwise comparisons or 1 -way ANOVA followed by Tukey's post hoc test for multiple comparisons. 
It has been previously reported that UDP-G augments migration of human neutrophils in Boyden chambers, and that PPTN inhibits this migration (20). However, in that study, the authors did not rule out the possibility of UDP-G-mediated chemokinesis, as opposed to chemotaxis. A different study showed that UDP-G can indirectly enhance neutrophil chemotaxis in the presence of a human endometrial epithelial cell line by inducing IL-8 (21). In that study, there was no direct effect of UDP-G alone on chemotaxis. Our current study found that UDP-G did not directly induce eosinophil chemotaxis, or induce production of eosinophil chemoattractants. On the contrary, levels of the eosinophil chemoattractant, CCL24, were even higher in $\mathrm{P} 2 \mathrm{ry} 14^{-/-}$mice than in wild-type mice. Although the explanation for this observation is not known, it is possible that a negative feedback mechanism exists for eosinophil recruitment, such that CCL24 production is suppressed in the setting of very strong eosinophilic inflammation. If so, CCL24 would be expected to be suppressed in wild-type mice undergoing strong eosinophilic inflammation, but not in $\mathrm{P} 2 \mathrm{ry} 14^{-/-}$mice that have lower eosinophilia.

In contrast to previously proposed mechanisms for UDP-Gmediated leukocyte recruitment, we found that this molecule promotes nondirectional chemokinesis because cell migration in the Boyden chamber was augmented when UDP-G was placed in both the top and bottom chambers, a setting in which there is no concentration gradient. Importantly, UDP-G also enhanced the ability of CCL24 to promote directional chemotaxis, suggesting that this is a likely mechanism by which UDP-G promotes eosinophil recruitment to the airway in the protease-mediated model of asthma. Thus, eosinophils are acted upon by IL-5 (22), UDP-G, and eotaxin, which promote proliferation, chemokinesis, and chemotaxis, respectively. It is possible that UDP-G might also affect responses to other chemotactic molecules, such as PGD2. The mechanism by which $\mathrm{P}_{2} \mathrm{Y}_{14} \mathrm{R}$ and the CCL24 receptor, CCR3, cooperate to amplify eosinophilic migration is currently unknown. However, there is precedent for different GPCRs to form heterodimers and augment strength of signaling (23-25). This potential for heterodimerization includes CCR3, which can act synergistically with the formyl peptide (fMLP) receptor 1 to induce ERK phosphorylation and cell chemotaxis (26). Thus, CCR3 might form a heterodimer with $\mathrm{P} 2 \mathrm{Y}_{14} \mathrm{R}$, although it is also possible that these 2 receptors act independently to promote chemotaxis and chemokinesis, respectively.

A potential caveat to experiments using UDP-G is that even very small amounts of endotoxin contamination can confound the interpretation of experimental findings (27). In agreement with this, we found that although instillation of UDP-G into the airways of mice induced rapid airway neutrophilia, this response was not seen in $\mathrm{Tlr} 4^{-/}$mice. This suggests that contaminating endotoxin was responsible for the observed neutrophilia, a conclusion supported by the finding that UDP-G-induced neutrophilia also occurred in $P 2 r y 14^{-/}$mice. By contrast, we found that UDP-Ginduced chemokinesis was seen for eosinophils of both wild-type and $T l r 4^{-/-}$mice, but not $P 2 r y 14^{-/-}$mice. We conclude from these experiments that the interaction of UDP-G with $\mathrm{P} 2 \mathrm{Y}_{14} \mathrm{R}$ promotes eosinophil chemokinesis.

Several previous studies have reported the ability of UDP-G to promote neutrophil chemotaxis ex vivo and the recruitment of these cells to various organs in vivo $(12,21)$. We also observed UDP-G-mediated neutrophil recruitment to the lung. However, in our hands this recruitment was also seen in $\mathrm{P} 2 \mathrm{ry} 14^{-/-}$mice, but not in $T l r 4^{-/-}$mice, suggesting that contaminating endotoxin is largely responsible for neutrophil recruitment. Although we found that neutrophils expressed P2ry14, their expression levels were approximately 10-fold lower than that of eosinophils. In addition, the amounts of UDP-G in the airways of OVA-challenged mice that had been previously sensitized through the airway using LPS/OVA were similar to those of untreated mice and far below the amounts seen in OVA-challenged mice previously sensitized using ASP/OVA. Similar findings were seen following extrapulmonary sensitization using alum as the adjuvant. It is possible that inhalation of protease during the sensitization phase predisposes epithelial cells to produce UDP-G upon challenge with OVA. It is likely that eosinophils, but not neutrophils, trigger the release of UDP-G into the airway, as supported by our adoptive transfer experiments using those 2 cell types. Taken together, our current data suggest that the UDP-G/P2Y ${ }_{14}$ R axis drives a positive feedback loop to promote eosinophilia.

Our current results are consistent with a recent study in which reanalysis of data from multiple gene expression studies identified multiple expression quantitative trait loci (eQTL) and an association between P2ry14 variants and asthma (28). That study also reported that UDP-G induces production of the potent type 2 cytokine, IL-33. This raises the possibility that UDP-G, acting through $\mathrm{P} 2 \mathrm{Y}_{14} \mathrm{R}$, can promote allergic sensitization. However, several experiments in our current study showed that P2ry14 is dispensable for allergic sensitization, and that some effects of UDP-G preparations are dependent on TLR4. It is nonetheless conceivable that UDP-G induction of IL-33 is relevant to the P2ry14enhanced eosinophil migration in vivo.

The impact of UDP-G and $\mathrm{P} 2 \mathrm{Y}_{14} \mathrm{R}$ on recruitment of different leukocyte subsets to inflamed tissue might be organ and disease dependent. Although we found no evidence supporting a role for this ligand-receptor pair in neutrophil recruitment to the lung during the animal models of asthma used here, a very recent study showed that $\mathrm{P} 2 \mathrm{Y}_{14} \mathrm{R}$ contributes to the initial stages of neutrophilic and monocytic inflammation in a mouse model of ischemic acute kidney injury (29). Some of these differences in cell recruitment between the models might reflect organ-specific differences in in P2ry14 expression. We found that both immature and mature eosinophils in the bone marrow express very low amounts of in P2ry14 RNA, whereas this gene was expressed very highly in lung eosinophils - even in the noninflamed lung. This suggests that the lung environment provides cues to eosinophils for upregulation of P2ry14. It will be interesting to learn whether other organs, such as the kidney, provide similar cues to other cell types, including neutrophils.

Finally, the ability of a small molecule inhibitor of $\mathrm{P} 2 \mathrm{Y}_{14} \mathrm{R}$ to suppress eosinophilic inflammation in vivo suggests that such molecules might have potential for therapeutic use in some asthma patients. Although early-onset eosinophilic forms of allergic asthma generally respond well to inhaled or oral glucocorticoids, these steroids have profound impacts on immunity, and their prolonged use is associated with many side effects, including yeast infections, cataracts, and osteoporosis. Other patients, particu- 
larly in the older age group, display type 2-high forms of eosinophilic asthma that are steroid resistant (5-7), but are responsive to antibodies against IL-5, IL-5R $\alpha$, IL-4R $\alpha$, IL-13, and IgE (30), and to leukotriene receptor antagonists (31). However, antibody-based treatments are very expensive, and there is an unmet need for novel approaches that can either stand alone, or be used as adjunctive therapy to allow lower doses of steroids or antibodies to be effectively used. Although we did not observe a difference in UDP-G levels between mild asthmatics and healthy volunteers (Karcz et al., unpublished observations), it is conceivable that levels of this molecule only increase during exacerbations, when eosinophilia rises (32). This would be analogous to the increases we observed for both UDP-G and eosinophils in mice following their challenge with allergen. Much work remains to be done on the mechanisms underlying the effects of $\mathrm{P} 2 \mathrm{Y}_{14} \mathrm{R}$ on immunity, but compounds that target this receptor might be useful as stand-alone or adjunctive therapies to treat some types of asthma. Recent progress in the use of noninvasive biomarkers to identify this class of patient should be extremely helpful in identifying individuals that are most likely to benefit from such an approach (7).

\section{Methods}

Mice. Male mice of the following mouse strains were purchased from The Jackson Laboratory: C57BL/6J, OT-II (B6.Cg-Tg[TcraTcrb]425Cbn/J), Tlr4 ${ }^{-/-}$(B6.129P2-Tlr4<tm1 Aki>), B6 Cd45.1 (B6. SJL-Ptprc ${ }^{\mathrm{a}}$ Pepc ${ }^{\mathrm{b}} /$ BoyJ), Cd11c-cre (B6.Cg-Tg[Itgax-cre]1-1Reiz/J), LyzM-cre (B6.129P2-Lyz2 ${ }^{\text {tm1(cre)lfo } / J), ~ a n d ~ C M V-c r e ~(B 6 . C-T g[C M V-~}$ cre]1Cgn/J). NJ.1638 (C57BL/6JJ-Tg[Cd3d-Il5]NJ.1638Nal) mice (33), hE2 (C57BL/6J-Tg[Scgb1a-hCCL24]hE2JLee) mice (34), and eoCre $\left(\mathrm{C} 57 \mathrm{BL} / 6 \mathrm{~J}-\mathrm{Epx}^{\mathrm{tm} 1.1(\mathrm{cre})) \mathrm{lee}}\right)$ mice (19) were obtained from Elizabeth A. Jacobsen (Mayo Clinic, Phoenix, Arizona, USA). The former 2 strains were intercrossed to generate double-transgenic mice with spontaneous airway eosinophilia (34). $C d 45.1 / C d 45.2$ heterozygous mice were generated by crossing C57BL/6J (Cd45.2) and B6 Cd45.1 animals. Conditionally mutant P2ry14 mice were obtained from EMMA (http://www.informatics.jax.org/strain/MGI:5905288). Crossing of $P 2 r y 14^{f / f l}$ mice with the $C M V$-cre deleter strain resulted in global disruption of the P2ry14 gene ( $P 2 r y 14^{-/-}$mice). In all experiments, ageand genetically matched mice from the same commercial source were used as controls. Mice were housed in specific pathogen-free conditions and used between 6 and 12 weeks of age.

Animal models of asthma. To sensitize mice, they were lightly anesthetized with isoflurane and given 2 oropharyngeal (o.p.) administrations, 1 week apart, of $50 \mu \mathrm{g}$ LPS-free OVA (Worthington Biochemical) together with an adjuvant: 100 ng LPS from E. coli 0111:B4 (SigmaAldrich), $20 \mu \mathrm{g}$ protease from Aspergillus oryzae (Sigma-Aldrich), or 10 $\mu \mathrm{g}$ protease from papaya (papain, Sigma-Aldrich) in a total volume of $50 \mu \mathrm{L}$ with PBS as the diluent. In some experiments, mice were sensitized by i.p. injection of $20 \mu \mathrm{g}$ LPS-free OVA complexed in Imject Alum Adjuvant (Thermo Fisher Scientific) in a total volume of 100 $\mu \mathrm{L}$ at the same schedule as in case of o.p. sensitizations. Seven days after the second sensitization, mice were challenged by exposing them to an aerosol of 1\% OVA (Sigma-Aldrich) in PBS for 1 hour. Unless otherwise indicated, BALF was collected 4 hours after challenge for analysis of cytokines in the airway, whereas airway inflammation and AHR were assessed 48 hours after challenge. PPTN hydrochloride was purchased from Tocris and was administered in a vehicle consisting of $10 \%$ DMSO, 30\% PEG-400, and $60 \%$ water. A dose of $10 \mathrm{mg} / \mathrm{kg}$ was selected, based on solubility considerations and efficacy in doseresponse experiments (Supplemental Figure 11). UDP-G was purchased from Cayman Chemical. A stock solution (12.5 mM) was freshly prepared in assay medium before each experiment.

AHR. Evaluations of AHR were performed as previously described (16), using the FlexiVent mechanical ventilator system (SCIREQ). A single compartment model of the lung was used to assess total respiratory system resistance after delivery of aerosolized methacholine using an ultrasonic nebulizer. Data are reported as peak resistance values.

Antibody responses. To assess antibody responses, venous blood was collected, and serum was separated using BD Microtainer Plasma Separator Tubes (BD Biosciences) and centrifugation at $6000 \mathrm{~g}$ for $5 \mathrm{~min}$ utes. To quantify OVA-specific IgE in serum, samples (diluted 1:2) were analyzed using the murine OVA-specific IgE ELISA Kit (BioLegend).

Histology. Lungs were fixed in $10 \%$ formalin and embedded in paraffin. Left lobe longitudinal sections 5 to $7 \mu \mathrm{m}$ thick were stained with Alcian blue and periodic acid-Schiff and scanned using the Aperio AT2 Digital Whole Slide Scanning system (Leica Biosystems). Images were generated using ImageScope software version 12.4.3 (Leica Biosystems). The mucin-producing cells were evaluated using FIJI (35). The RGB images were resized, then split into red, green, and blue images. The green image was used to set a threshold and make a mask, the Fill Holes and Watershed command was run to improve the image, and the Analyze Particles command used to count cells.

Analysis of cytokines in BALF and regional LNs. Concentrations of cytokines and chemokines in BALF were measured as described previously (16), using a multiplexed fluorescent bead-based immunoassay according to the instructions of the manufacturer (Bio-Rad Laboratories). To indirectly study $\mathrm{T}$ cell development in lung-draining mLNs, Histopaque gradient-enriched cells were prepared from spleens and LNs of OT-II transgenic mice as previously described (36), washed 3 times with sterile PBS, and $1 \times 10^{7}$ cells transferred by tail vein injection into recipient mice. These animals were then given o.p. administrations of OVA together with a test adjuvant. Four days after sensitization, the mLNs were excised, minced, pressed through a 70 $\mu \mathrm{m}$ strainer, and $1 \times 10^{6}$ cells cultured in $200 \mu \mathrm{L}$ cRPMI media $(10 \%$ fetal bovine serum, 0.1\% 2-mercaptoethanol, $10 \mathrm{mM}$ Hepes, and 100 $\mathrm{U} / \mathrm{mL}$ penicillin/streptomycin) containing $10 \mu \mathrm{g} / \mathrm{mL}$ OVA for 2 days. Supernatants were analyzed for cytokines using multiplexed fluorescent bead-based immunoassays.

Bone marrow-chimeric mice. Bone marrow-chimeric mice were generated by using C57BL/6J, P2ry14 $4^{-/}$, or heterozygous $\mathrm{Cd} 45.1 / \mathrm{Cd} 45.2$ mice as recipients. Bone marrow cells $\left(1 \times 10^{7}\right)$ from C57BL/6J, P2ry $14^{-/-}$, or mixed Cd45.1/P2ry14 ${ }^{-/-}$(Cd45.2) (1:1) were transferred by means of tail vein injection into lethally irradiated recipient animals ( 9 Gy over 15 minutes). Before their use in experiments, chimeric mice were rested for 10 to 12 weeks to allow for hematopoietic reconstitution.

Preparation of IL-5- and CCL24-induced lung eosinophils. Mice were given $100 \mathrm{ng}$ recombinant mouse (rm) IL-5 (Peprotech) by tail vein injection on 4 consecutive days. Four hours after the last injection, the animals were given o.p. administrations of $1 \mu \mathrm{g}$ rmIL-5 and $3 \mu \mathrm{g}$ rmCCL24 (Peprotech) in $50 \mu \mathrm{L}$ of PBS. Lung eosinophils were sorted by flow cytometry 10 hours later, and RNA was prepared from them.

Cell preparations from lung tissue, bone marrow, and blood. Lungs were perfused by PBS injection into the right ventricle. For preparation of DCs, macrophages, and monocytes, minced tissues were digested 
for 60 minutes at $37^{\circ} \mathrm{C}$ with $100 \mu \mathrm{g} / \mathrm{mL}$ Liberase, $125 \mathrm{U} / \mathrm{mL}$ collagenase XI, $60 \mathrm{U} / \mathrm{mL}$ hyaluronidase 1a, and $200 \mu \mathrm{g} / \mathrm{mL}$ DNase I (all from Sigma-Aldrich), as described previously (37). To prepare other lung leukocytes, a similar protocol was followed, except that the lung was digested for 30 minutes. Single-cell suspensions were passed through a $70 \mu \mathrm{m}$ strainer. To enrich DCs and lung macrophages, low-density cells in the lung cells were collected by gradient centrifugation using $16 \%$ Nycodenz (Accurate Chemical \& Scientific). Lymphocytes, monocytes, and granulocytes were enriched by gradient centrifugation using 2 layers of gradient solutions: $14.5 \%$ Nycodenz and Histopaque 1119 (Sigma-Aldrich) and collection of the higher-density cell layer at the interface of the Histopaque 1119 and cell suspension buffer.

Preparation of alveolar epithelial cells (AECs) and lung endothelial cells was performed as described elsewhere (38). Briefly, PBSperfused lungs were excised and inflated with $4 \mathrm{U} / \mathrm{mL}$ elastase (Worthington Biochemical), $1 \mathrm{U} / \mathrm{mL}$ Dispase (Sigma-Aldrich), and $200 \mu \mathrm{g} / \mathrm{mL}$ DNase I for 45 minutes at $37^{\circ} \mathrm{C}$. Lungs were then minced and tissue fragments were filtered through a $70 \mu \mathrm{m}$ strainer. Lung pieces retained on the strainer were digested with $25 \mu \mathrm{g} / \mathrm{mL}$ Liberase and $200 \mu \mathrm{g} / \mathrm{mL}$ DNase I for an additional 30 minutes at $37^{\circ} \mathrm{C}$ and the dissociated tissue was combined with cells released in first digestion step. The resulting cells were incubated with biotinylated anti-CD45 mAb (30-F11, BD Biosciences) and anti-TER-119 mAb (TER119, BD Biosciences). Following the incubation of antibody-labeled cells with Streptavidin RapidSpheres, an EasySep magnet (Stemcell Technologies) was used to deplete leukocytes and red blood cells (RBCs).

Bone marrow cells were collected from pulverized sternums, femurs, tibias, and humeri. RBCs were lysed with ACK lysis buffer (150 $\mathrm{mM} \mathrm{NH}_{4} \mathrm{Cl}, 10 \mathrm{mM} \mathrm{KHCO}_{3}, 0.1 \mathrm{mM} \mathrm{Na}_{2}$ EDTA) and the obtained cells were used without further enrichment.

Blood was collected from the inferior vena cava. Cells from heparinized blood were centrifuged on a Histopaque 1083 gradient (Sigma-Aldrich). Leukocytes were obtained following RBC lysis by hypo-osmotic shock in distilled water.

Flow cytometry and purification of cell populations. Cells were blocked with anti-mouse CD16/anti-mouse CD32 (2.4G2) and normal mouse and rat serum (Jackson ImmunoResearch). For staining of surface antigens, cells were incubated with one or more of the following fluorochrome-conjugated (allophycocyanin-Cy7 [APC-Cy7], Alexa Fluor [AF] 488, AF 647, Brilliant Violet [BV] 510, BV 711, Brilliant Ultra Violet [BUV] 395, BUV 737, eFluor 450, FITC, PerCP-Cy5.5, phycoerythrin [PE], and PE-Dazzle 594) or biotin-conjugated antibodies against mouse CCR3 (83101, R\&D Systems), CD3e (clone 145-2C11), CD11b (clone M1/70), CD11c (clone N418 and HL3), CD19 (clone 605), CD31 (clone 390), CD45 (clone 30-F11), CD45.1 (clone A20), CD45.2 (clone 104), CD88 (clone 20/70), CD103 (clone M290), CD115 (clone AFS98), CD125 (clone T21), CD326 (clone G8.8), Ly-6C (clone AL-21), Ly-6G (clone 1A8), MHC class II I-A ${ }^{b}$ (clone AFb.120), and Siglec-F (clone E50-2440) (BD Biosciences, BioLegend, or eBioscience). Incubation with biotinylated antibodies was followed by staining with fluorochrome-conjugated streptavidin. Stained cells were analyzed on an LSRFortessa flow cytometer (BD Biosciences), and the data were analyzed using FACSDiva (BD Biosciences) and FlowJo software (Tree Star). Only single cells were analyzed, and dead cells were excluded based on 7-aminoactinomycin D (7AAD) (BD Biosciences) or eFluor 780 Fixable Viability Dye (eBioscience) staining. Where applicable, apoptotic cells were identified based on FITC-conjugated annexin
$\mathrm{V}$ staining (BD Biosciences). Cell subtypes were identified based on the following staining patterns: neutrophils $\left(\mathrm{CD} 45^{+} \mathrm{CD} 11 \mathrm{c}^{\mathrm{lo}} \mathrm{MHC}-\mathrm{II}^{\mathrm{lo}}\right.$

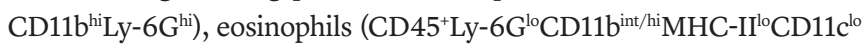
Siglec- $\left.\mathrm{F}^{+} \mathrm{CCR} 3^{+}\right)$, alveolar macrophages $\left(\mathrm{CD} 45^{+} \mathrm{CD} 11 \mathrm{~b}^{\mathrm{lo}} \mathrm{Ly}-6 \mathrm{G}^{\mathrm{lo}}\right.$ autofluorescence ${ }^{\text {hi }} \mathrm{CD} 11 \mathrm{c}^{\text {hi }} \mathrm{CD} 88^{\text {int }}$ Siglec- $\mathrm{F}^{\text {hi }}$ ), lung interstitial macrophages $\left(\mathrm{CD} 45^{+} \mathrm{CD} 11 \mathrm{~b}^{\text {hi }} \mathrm{Ly}-6 \mathrm{G}^{\mathrm{lo}} \mathrm{CD} 11 \mathrm{c}^{\text {int }} \mathrm{CD} 88^{\text {hi }}\right.$ Siglec-F $\left.\mathrm{F}^{\mathrm{lo}}\right), \mathrm{CD}_{11 \mathrm{~b}^{+}} \quad \mathrm{cDCs}$ (CD $45^{+}$Ly- $6 \mathrm{G}^{\text {lo }}$ autofluorescence ${ }^{\mathrm{lo}}$ Siglec- $\mathrm{F}^{\mathrm{lo}} \mathrm{CD} 88^{\mathrm{lo}} \mathrm{CD} 11 \mathrm{c}^{\text {hi }} \mathrm{MHC}-\mathrm{II}^{\mathrm{hi}}$ $\left.\mathrm{CD} 103^{\text {lo }} \mathrm{CD} 11 \mathrm{~b}^{\mathrm{hi}}\right), \mathrm{CD} 103^{+} \mathrm{cDCs} \quad\left(\mathrm{CD} 45^{+} \mathrm{Ly}-6 \mathrm{G}^{\mathrm{lo}}\right.$ autofluorescence ${ }^{\text {lo }}$ Siglec- $\left.-\mathrm{F}^{\mathrm{lo}} \mathrm{CD} 88^{\mathrm{lo}} \mathrm{CD} 11 \mathrm{c}^{\text {hi }} \mathrm{MHC}-\mathrm{II}^{\text {hi }} \mathrm{CD} 11 \mathrm{~b}^{\text {lo }} \mathrm{CD} 103^{+}\right)$, lung monocytes $\left(\mathrm{CD} 45^{+} \mathrm{CD} 11 \mathrm{~b}^{\text {hi }}\right.$ Ly- $6 \mathrm{G}^{\text {lo }}$ autofluorescence ${ }^{\text {hi }}$ Siglec- $-\mathrm{F}^{\mathrm{lo}} \mathrm{CD} 88^{\mathrm{lo}} \mathrm{MHC}-\mathrm{II}^{\mathrm{lo}}$ CD $115^{\text {hi }}$ Ly-6C $\left.{ }^{\text {hi }}\right)$, lung lymphocytes $\left(C D 45^{+}\right.$Ly- $6 \mathrm{G}^{\text {lo }}$ Siglec $-F^{\text {lo }} C D 11 c^{\text {lo }}$ $\left.\mathrm{CD} 88^{\text {lo }} \mathrm{CD} 115^{\text {lo }} \mathrm{CD} 3 / \mathrm{CD} 19^{+}\right)$, lung epithelial cells $\left(\mathrm{CD} 45^{\text {lo }} \mathrm{CD} 326^{\mathrm{hi}}\right)$, and lung endothelial cells $\left(\mathrm{CD} 45^{\mathrm{lo}} \mathrm{CD} 31^{\mathrm{hi}}\right)$. Purification of individual cell types from tissue was performed using flow cytometric sorting on a BD FACSAria instrument, as previously described (39). Purity of the isolated cells was consistently greater than $95 \%$.

Identification of marginated vascular cells. PCLS were generated as described previously (40) and stained using antibodies against CCR3 (eosinophils), CD31 (endothelial cells), and CD324 (epithelial cells). Mean distances from eosinophils to the nearest endothelial cell $(n=$ 5000) was calculated using Visiopharm Oncotopix software (Visiopharm Corporation).

For flow cytometry-based analysis of marginated cells, BV 711labeled anti-mouse CD45.2 antibodies (clone 104, BD Biosciences) were injected i.v. at $1 \mu \mathrm{g} / \mathrm{mouse}, 5$ minutes prior to sacrifice. Lungs were perfused, excised, washed in $5 \mathrm{~mL}$ PBS, and processed as described above. Cells labeled with BV 711-conjugated antibodies were identified in perfused lungs as the marginated vascular cell population.

Gene expression analysis. Total RNA was extracted using TRIzol (Thermo Fisher Scientific) and converted to cDNA using oligo(dT) primers and a SuperScript III First-Strand kit (Thermo Fisher Scientific). Quantitative PCR amplification was performed using SYBR Green Master Mix (Thermo Fisher Scientific) on an Mx3000P QPCR system (Agilent Technologies) using the following primers: P2ry14 forward, CCCAGAAGAGAGAAAATCTTTG; P2ry14 reverse, CTGATATCCCATTGAGGAGG; Ppih forward, CGgAGAGTTCAGAAAAGATG; and Ppih reverse, CCATCGCCATTAACAAAATC. Relative expression of the P2ry14 gene was normalized to that of the housekeeping gene Ppih (peptidyl-prolyl cis-trans isomerase $\mathrm{H}$ ).

RNA-seq analysis. RNA of lung eosinophils collected 16 hours after OVA challenge from OVA/ASP-sensitized C57BL/6J or P2ry $14^{-/-}$mice was isolated using TRIzol Plus reagent, followed by DNase treatment and additional cleanup step using a PureLink RNA Mini kit (Thermo Fisher Scientific) according to the manufacturer's instructions. Unstranded RNA-seq libraries with unique barcode adapters were constructed from total RNA using a TruSeq RNA sample prep kit (Illumina) according to the manufacturer's instructions. Multiplexed libraries were sequenced by the National Institute of Environmental Health Sciences Epigenomics and DNA Sequencing Core Laboratory on a NovaSeq 6000 (Illumina) as single-end 76-mers. Raw reads were filtered to retain only those with a mean base quality score greater than 20. Filtered reads were mapped to the mm10 reference genome via STAR (41) version 2.5 (parameters --outMultimapperOrder Random --outSAMattrIHstart 0 --outFilterType BySJout --alignSJoverhangMin 8 --limitBAMsortRAM 55000000000 --outSAMstrandField intronMotif --outFilterIntronMotifs RemoveNoncanonical). Subread featureCounts (42) v1.5.0-p1 (parameters: -s0) was used to 
collect counts per gene per sample for a set of gene models defined by RefSeq transcripts as downloaded from the UCSC Table Browser (http://genome.ucsc.edu/cgi-bin/hgTables) as of April 27, 2018. Differentially expressed genes were identified with DESeq2 (43) v1.14.1 at an FDR threshold of 0.05. The volcano plot was generated based on $-\log _{10}$-adjusted $P$ values and $\log _{2}$ (fold change) values as reported by DESeq2. The expression heatmap was generated using $\mathrm{R}$ function heatmap.2 with row-scaling DESeq2's rlog-transformed scores. The list of genes for generation of the expression heatmap was created based on the Nanostring nCounter Myeloid Innate Immunity V2 Panel (Nanostring Technologies) through filtration for records annotated as eosinophil-associated genes. The RNA-seq data have been submitted to the NCBI's Gene Expression Omnibus database (GEO GSE150347).

Adoptive transfer of granulocytes to the airways. To obtain neutrophils, C57BL/6J mice were treated by o.p. instillation of $0.35 \mu \mathrm{g}$ recombinant mouse CXCL1 (KC, R\&D Systems) together with 0.35 $\mu \mathrm{g}$ recombinant mouse CXCL5 chemokine (LIX, R\&D Systems) in 50 $\mu \mathrm{L}$ of PBS. BALF cells were collected after 16 hours and were found to be greater than $85 \%$ neutrophils. Eosinophils were collected in BALF from naive, untreated hE2 $\times$ NJ.1638 mice (>90\% eosinophils). Cell samples containing neutrophils or eosinophils were washed twice and resuspended in PBS. Cell density was adjusted to $4 \times 10^{6}$ cells $/ \mathrm{mL}$ and the obtained cell suspension was instilled to the airways of C57BL/6J mice at $2 \times 10^{5}$ cells per mouse. BALF was collected after 4 hours and analyzed for UDP-hexose levels.

Depletion of eosinophils. Eosinophils were depleted by i.p. injections of $150 \mu \mathrm{g}$ anti-mouse CCR3 antibodies (clone 6S2-19-4, Bio X Cell) on 3 consecutive days. The isotype control used in depletion experiments was rat IgG2b (clone LTF-2, Bio X Cell). Flow cytometry was used to assess depletion of the eosinophils 24 hours after the last antibody injection.

Mass spectrometric sample preparation and analysis of UDP-hexose in BALF. ${ }^{13} \mathrm{C}_{6}-\mathrm{UDP}-\mathrm{G}$ (Cambridge Isotope Laboratories) was added to $\mathrm{PBS}$, so that the final concentration was $30 \mathrm{fmol} / \mu \mathrm{L}$. One milliliter of this solution was used to perform BAL. Cells in BALF were obtained by centrifugation for 5 minutes at $300 \mathrm{~g}$ and $4^{\circ} \mathrm{C}$. Supernatants were transferred to fresh $1.5 \mathrm{~mL}$ tubes and incubated for 2 minutes at $95^{\circ} \mathrm{C}$. After cooling to $4^{\circ} \mathrm{C}$, samples were centrifuged again for 10 minutes at $17,000 \mathrm{~g}$ and $4^{\circ} \mathrm{C}$. Debris was discarded, and cleared BALF was stored at $-80^{\circ} \mathrm{C}$ for further analysis. Solid-phase extraction (SPE) was then performed on $500 \mu \mathrm{L}$ of the lavage samples using Hypercarb Hypersep $25 \mathrm{mg}$ cartridges (Thermo Fisher Scientific) and a positive pressure manifold with $\mathrm{N}_{2}$ gas as the head pressure. The SPE cartridges were first preconditioned by passing $1 \mathrm{~mL}$ of $0.3 \%$ formic acid in 40:60 water/acetonitrile ( $\mathrm{pH} 9.0$ ) through the cartridges and then equilibrating twice with $0.5 \mathrm{~mL}$ water. After sample loading, $0.5 \mathrm{~mL}$ water was passed over the cartridges 3 times followed by an elution with 2 times $0.3 \mathrm{~mL} \mathrm{0.3 \%} \mathrm{formic} \mathrm{acid} \mathrm{in} \mathrm{40:60} \mathrm{water/acetonitrile} \mathrm{(} \mathrm{pH}$ 9.0). The eluants were dried to completion using a CentriVap Concentrator (Labconco) and then resuspended in $20 \mu \mathrm{L}$ of water prior to LC-MS analyses. Five microliters of the sample was injected onto the column for liquid chromatography-mass spectrometry (LC-MS) analysis. Data were acquired on a Q Exactive Plus mass spectrometer (QE-MS, Thermo Fisher Scientific) interfaced with a Vanquish (Thermo Fisher Scientific) UHPLC system. Reverse-phase chromatography was performed using a CORTECS C18 column $(100 \times 2.1 \mathrm{~mm}$ i.d., 1.6 $\mu \mathrm{m}$ particle size; Waters Corporation) and a CORTECS C18 VanGuard precolumn $(5 \times 2.1 \mathrm{~mm}$ i.d., $1.6 \mu \mathrm{m}$ particle size; Waters Corporation) with solvent A being $5 \mathrm{mM}$ ammonium formate in water ( $\mathrm{pH}$ 6.5) and solvent B being methanol and a flow rate of $150 \mu \mathrm{L}$ per minute. The LC gradient included a ramp from $0 \%$ to $42 \%$ B from 0 to 6 minutes followed by a ramp from $42 \%$ to $95 \%$ B over 1 minute and then a 3-minute hold at $95 \%$ B. The run was completed with a ramp of $95 \%$ to $0 \% \mathrm{~B}$ for 0.5 minutes followed by a 4.5 -minute recondition at $0 \%$ B. The QE-MS was equipped with a HESI source used in the negative ion mode cycling through a single MS scan followed by 2 PRM scans (UDP-hexose $m / z 565.05 ;{ }^{13} \mathrm{C}_{6}$-UDP-G standard $m / z$ 571.07) using a normalized collision energy of 30 for both PRM scans and with the following instrument parameters: sheath gas, 10; auxiliary gas, 5; sweep gas, 1 ; spray voltage, $3.5 \mathrm{kV}$; capillary temperature, $325^{\circ} \mathrm{C}$; S-lens, 50 ; scan range $(\mathrm{m} / \mathrm{z})$ of 200 to $1000 ; 2-\mathrm{m} / z$ isolation window; resolution, 17,500; automated gain control (AGC), $2 \times 10^{5}$ ions; and a maximum IT of $200 \mathrm{~ms}$. Mass calibration was performed before data acquisition using the Pierce ESI Negative Ion Calibration Mixture (Thermo Fisher Scientific). Data were processed using the Qual Browser application in the Xcalibur software suite (Thermo Fisher Scientific) in which extracted ion chromatograms were generated from the PRM channels using the peak area for $m / z 323.0$ for quantification in both channels and the ion $m / z 241.0$ for qualification of the endogenous UDP-hexose and the ion $m / z 247.0$ for qualification of the ${ }^{13} \mathrm{C}_{6}$-labeled UDP-G internal standard. The ratio of the areas of the endogenous UDP-hexoses to the UDP-G internal standard is reported.

In vitro migration assay. BALF cells were collected 48 hours after allergen challenge. Following RBC lysis, cells were resuspended in RPMI medium (Thermo Fisher Scientific) supplemented with $0.5 \%$ bovine serum albumin (BSA) (Sigma-Aldrich), and left to equilibrate at $37^{\circ} \mathrm{C}$ for 15 minutes. A cell suspension $\left(2 \times 10^{5}\right.$ cells in $\left.100 \mu \mathrm{L}\right)$ was added to the upper compartment of a Transwell cell culture chamber (pore size, $5.0 \mu \mathrm{m}$ ) (Corning Costar) on a 24-well plate containing 600 $\mu \mathrm{L}$ of $0.5 \%$ BSA in RPMI with or without $30 \mu \mathrm{M}$ UDP-G and/or $50 \mathrm{ng} /$ $\mathrm{mL}$ CCL24 (Peprotech). In some cases, $30 \mu \mathrm{M}$ UDP-G was added also together with the cells to the upper compartment of the Transwell chamber. After incubation at $37^{\circ} \mathrm{C}$ for 2 hours, migrated cells were collected from the bottom compartment and analyzed by flow cytometry. Migrated eosinophils were identified based on FSC $\times$ SSC gating and their number was determined using CountBright Absolute Counting Beads (BD Biosciences). The experiments were performed in triplicate and the numbers of migrated eosinophils were normalized to the mean counts of cells that spontaneously migrated in medium alone.

Statistics. Most statistical calculations were performed using GraphPad Prism 8. Data are shown as means \pm SEM. Unless indicated otherwise, results shown represent data from at least 2 independent experiments giving similar results. Differences between groups were identified by ordinary 1-way analysis of variance (ANOVA) with Tukey's multiple-comparison test. Individual comparisons between groups were confirmed by a 2-tailed Student's $t$ test or 1-way ANOVA followed by Bonferroni's post hoc test. Where appropriate, outliers were removed based on the ROUT method (44) in GraphPad Prism, using a $Q$ parameter setting of $5 \%$. $P$ values of less than 0.05 were considered statistically significant.

Data and materials availability: The RNA-seq data have been submitted to the NCBI's Gene Expression Omnibus (https://www.ncbi. nlm.nih.gov/geo/) and can be found under the GSE150347 study at: https://www.ncbi.nlm.nih.gov/geo/query/acc.cgi?acc=GSE150347. 
Study approval. All animal studies were reviewed and approved by the Institutional Animal Care and Use Committee at the National Institute of Environmental Health Sciences, Research Triangle Park, North Carolina.

\section{Author contributions}

KAJ and DNC conceived of the project. TPK, KAJ, HN, and DNC analyzed data and designed experiments with cells and animals. TPK and GSW performed experiments with cells and animals. JGW and LJD designed and performed experiments to analyze UDP-sugars in BALF. KN generated libraries for RNA-seq. SAG performed RNA-seq data analysis. TPK and DNC wrote the manuscript, and all authors made editorial suggestions and approved the final version.

\section{Acknowledgments}

We thank Ligon Perrow for support with animal experiments; Erica Scappini and C. Jeffery Tucker for analysis of histologic sections; Elizabeth Jacobsen (Mayo Clinic) and Helene Rosenberg
(NIAID/NIH) for NJ.1638, hE2, and eoCRE mice; Maria Sifre and Carl Bortner for help with flow cytometry and cell sorting; Molly Cook and Jason Malphurs and for help with RNA-seq; Norris Flagler for analysis of PCLS; and Michael Fessler and Jennifer Martinez for critical reading of the manuscript. This work was supported by the Intramural Research Program of the NIH, the National Institute of Environmental Health Sciences (ZIA ES102025-13), and the National Institute of Diabetes and Digestive and Kidney Diseases (ZIA DK31117).

Address correspondence to: Donald N. Cook, Immunogenetics Group, Immunity, Inflammation and Disease Laboratory, 111 T.W. Alexander Drive, Building 101, D2-01, Research Triangle Park, North Carolina 27709, USA. Phone: 984.287.4245; Email: cookd@niehs.nih.gov.

TPK's present address is: Department of Technology and Biotechnology of Drugs, Jagiellonian University Medical College, 30-688 Kracow, Poland.
1. Martinez FD, Vercelli D. Asthma. Lancet. 2013;382(9901):1360-1372.

2. Lambrecht BN, Hammad H. The immunology of asthma. Nat Immunol. 2015;16(1):45-56.

3. Lambrecht BN, et al. The cytokines of asthma. Immunity. 2019;50(4):975-991.

4. Bello CE, Garrett SD. Therapeutic issues in oral glucocorticoid use. Lippincotts Prim Care Pract. 1999;3(3):333-341.

5. Kabata $\mathrm{H}$, et al. Thymic stromal lymphopoietin induces corticosteroid resistance in natural helper cells during airway inflammation. Nat Commun. 2013;4:2675.

6. Liu S, et al. Steroid resistance of airway type 2 innate lymphoid cells from patients with severe asthma: the role of thymic stromal lymphopoietin. J Allergy Clin Immunol. 2018;141(1):257-268.

7. Peters MC, et al. Refractory airway type 2 inflammation in a large subgroup of asthmatic patients treated with inhaled corticosteroids. JAllergy Clin Immunol. 2019;143(1):104-113.

8. Iwasaki A, Medzhitov R. Regulation of adaptive immunity by the innate immune system. Science. 2010;327(5963):291-295.

9. Matzinger $P$. The danger model: a renewed sense of self. Science. 2002;296(5566):301-305.

10. Lazarowski ER, et al. Release of cellular UDPglucose as a potential extracellular signaling molecule. Mol Pharmacol. 2003;63(5):1190-1197.

11. Le TT, et al. Purinergic signaling in pulmonary inflammation. Front Immunol. 2019;10:1633.

12. Sesma JI, et al. UDP-glucose promotes neutrophil recruitment in the lung. Purinergic Signal. 2016;12(4):627-635.

13. Whitehead GS, et al. TNF is required for TLR ligand-mediated but not protease-mediated allergic airway inflammation. J Clin Invest. 2017;127(9):3313-3326.

14. Walker JA, McKenzie ANJ. $\mathrm{T}_{\mathrm{H}} 2$ cell development and function. Nat Rev Immunol. 2018;18(2):121-133.

15. Eisenbarth SC, et al. Lipopolysaccharideenhanced, toll-like receptor 4-dependent T helper cell type 2 responses to inhaled antigen. J Exp
Med. 2002;196(12):1645-1651.

16. Wilson RH, et al. Allergic sensitization through the airway primes Th17-dependent neutrophilia and airway hyperresponsiveness. Am J Respir Crit Care Med. 2009;180(8):720-730.

17. Abram CL, et al. Comparative analysis of the efficiency and specificity of myeloid-Cre deleting strains using ROSA-EYFP reporter mice. J Immunol Methods. 2014;408:89-100.

18. Caton ML, et al. Notch-RBP-J signaling controls the homeostasis of CD8- dendritic cells in the spleen. J Exp Med. 2007;204(7):1653-1664.

19. Doyle AD, et al. Homologous recombination into the eosinophil peroxidase locus generates a strain of mice expressing Cre recombinase exclusively in eosinophils. J Leukoc Biol. 2013;94(1):17-24.

20. Barrett MO, et al. A selective high-affinity antagonist of the P2Y14 receptor inhibits UDP-glucose-stimulated chemotaxis of human neutrophils. Mol Pharmacol. 2013;84(1):41-49.

21. Arase T, et al. The UDP-glucose receptor P2RY14 triggers innate mucosal immunity in the female reproductive tract by inducing IL-8. J Immunol. 2009;182(11):7074-7084.

22. Dougan M, et al. GM-CSF, IL-3, and IL-5 family of cytokines: regulators of inflammation. Immunity. 2019;50(4):796-811.

23. Mellado M, et al. Chemokine receptor homo- or heterodimerization activates distinct signaling pathways. EMBO J. 2001;20(10):2497-2507.

24. Mustafa S, et al. Identification and profiling of novel $\alpha 1 \mathrm{~A}$-adrenoceptor-CXC chemokine receptor 2 heteromer. J Biol Chem. 2012;287(16):12952-12965.

25. Salanga CL, et al. Modulation of chemokine receptor activity through dimerization and crosstalk. Cell Mol Life Sci. 2009;66(8):1370-1386.

26. Gouwy M, et al. Chemokines and other GPCR ligands synergize in receptor-mediated migration of monocyte-derived immature and mature dendritic cells. Immunobiology. 2014;219(3):218-229.

27. Brautigam VM, et al. The inflammatory effects of UDP-glucose in N9 microglia are not mediated by P2Y14 receptor activation. Purinergic Signal. 2008;4(1):73-78.

28. Ferreira MA, et al. Gene-based analysis of regulatory variants identifies 4 putative novel asthma risk genes related to nucleotide synthesis and signaling. J Allergy Clin Immunol. 2017;139(4):1148-1157.

29. Battistone MA, et al. Pro-inflammatory P2Y14 receptor inhibition protects against ischemic acute kidney injury in mice. J Clin Invest. 2020;130(7):3734-3749.

30. Patel SS, et al. Biological therapies for eosinophilic asthma. Expert Opin Biol Ther. 2018;18(7):747-754.

31. Montuschi P, Peters-Golden ML. Leukotriene modifiers for asthma treatment. Clin Exp Allergy. 2010;40(12):1732-1741.

32. Nakagome K, Nagata M. Involvement and possible role of eosinophils in asthma exacerbation. Front Immunol. 2018;9:2220.

33. Lee NA, et al. Expression of IL-5 in thymocytes/T cells leads to the development of a massive eosinophilia, extramedullary eosinophilopoiesis, and unique histopathologies. JImmunol. 1997;158(3):1332-1344.

34. Ochkur SI, et al. Coexpression of IL-5 and eotaxin-2 in mice creates an eosinophil-dependent model of respiratory inflammation with characteristics of severe asthma. JImmunol. 2007;178(12):7879-7889.

35. Schindelin J, et al. Fiji: an open-source platform for biological-image analysis. Nat Methods. 2012;9(7):676-682.

36. Nakano H, et al. Pulmonary CD103(+) dendritic cells prime $\mathrm{Th} 2$ responses to inhaled allergens. Mucosal Immunol. 2012;5(1):53-65.

37. Nakano H, Cook DN. Pulmonary antigen presenting cells: isolation, purification, and culture. Methods Mol Biol. 2013;1032:19-29.

38. Nakano H, et al. Isolation and purification of epithelial and endothelial cells from mouse lung. Methods Mol Biol. 2018;1799:59-69.

39. Nakano H, et al. Complement receptor C5aR1/ CD88 and dipeptidyl peptidase-4/CD26 define distinct hematopoietic lineages of dendritic cells. 
J Immunol. 2015;194(8):3808-3819.

40. Lyons-Cohen MR, et al. Precision-cut mouse lung slices to visualize live pulmonary dendritic cells. J Vis Exp. 2017;(122):55465.

41. Dobin A, et al. STAR: ultrafast universal RNA-seq aligner. Bioinformatics. 2013;29(1):15-21.
42. Liao Y, et al. featureCounts: an efficient general purpose program for assigning sequence reads to genomic features. Bioinformatics. 2014;30(7):923-930.

43. Love MI, et al. Moderated estimation of fold change and dispersion for RNA-seq data with
DESeq2. Genome Biol. 2014;15(12):550.

44. Motulsky HJ, Brown RE. Detecting outliers when fitting data with nonlinear regression - a new method based on robust nonlinear regression and the false discovery rate. BMC Bioinformatics. 2006;7:123. 